\title{
Individualisierte Plattformarbeit und kollektive
}

Interessenartikulation

Fabian Hoose und Thomas Haipeter

\section{Einleitung}

Welche Ansatzpunkte bietet Plattformarbeit für die Entwicklung kollektiver Interessen der Beschäftigten? Unter welchen Bedingungen artikulieren Plattformbeschäftigte gemeinsame Interessen? Welche Beispiele lassen sich dafür finden? Und was lässt sich aus diesen Beispielen lernen? Diese Fragen sind für die aktuelle Diskussion um die Zukunft der Gewerkschaften und der Institutionen der deutschen Arbeitsbeziehungen von einiger Bedeutung. Denn viel spricht dafür, dass sie als Ausdruck der Individualisierung der Arbeitsbedingungen und der Digitalisierung von Tätigkeiten ein Menetekel sind für den strukturellen Wandel durch Digitalisierung, der die entwickelten Volkswirtschaften in den nächsten Jahren erfassen könnte (Schramm und Tietgen-Simonsen 2019). Die Fähigkeit der Artikulation kollektiver Beschäftigteninteressen auf diesem Terrain kann deshalb als eine der wichtigsten Vorbedingungen dafür betrachtet werden, dass Gewerkschaften, Betriebsräte und die mit ihnen verbundenen Institutionen der Arbeitsbeziehungen auch in der digitalen Arbeitswelt eine Zukunft haben.

Der Einsatz digitaler Plattforminfrastrukturen bei der Organisation und Verteilung von Arbeit verändert traditionelle Beschäftigung und lässt darüber hinaus auch gänzlich neue Formen von Erwerbsarbeit entstehen. Durch diese Entwicklungen wird mitunter eine Verschlechterung von Arbeitsbedingungen befürchtet. Dies ist nicht zuletzt deswegen der Fall, weil die Einführung bzw. Anwendung digitaler Technologien bei der Arbeit als vor allem durch Arbeitgeber forcierte Rationalisierungsstrategien angesehen werden. Arbeitnehmer*innen sehen sich durch den Einsatz digitaler Technologien bei der Ausführung ihrer Arbeit damit konfrontiert, dass der Arbeitsprozess und damit ihr Handeln während der Arbeit vollständig überwacht und aufgezeichnet wird und es zudem durch die gesteigerten Möglichkeiten der Datenverarbeitung auch zu einer Vereinzelung der Arbeitnehmerbetrachtung durch den Arbeitgeber kommt (Wedde 2017). Diese Informationsvorsprünge der Arbeitgeber durch die Analyse von Ar- 
beitnehmerdaten kann, so eine Befürchtung, dafür genutzt werden, um verstärkt Wettbewerbssituationen zwischen Arbeitnehmer*innen zu kreieren, durch die die Formulierung kollektiver Arbeitnehmerinteressen zunehmend erschwert wird.

„Plattformunternehmen“ (Kirchner und Beyer 2016, S. 329) vermögen es, Wertschöpfungsketten und Märkte neu zu organisieren oder gänzlich neue Felder und Branchen zu definieren, indem sie über Online-Infrastrukturen Anbieter und Nachfrager miteinander vernetzen und hierfür Provisionsgebühren erheben. Indem sie so neue Märkte schaffen oder vorhandene Märkte verändern, kommt es zu einer zunehmenden Individualisierung insbesondere auf der Anbieterseite von Arbeitstätigkeiten. Die digitale Koordination über Plattformen erlaubt es, arbeitsteilige Produktion über Unternehmensgrenzen hinaus effizient zu gestalten. In der Folge sind hier immer mehr Solo-Selbstständige ohne feste Anbindung an einzelne Unternehmen tätig. Mit der Etablierung digitaler, internetbasierter Plattformunternehmen treten dabei im Austauschprozess von Arbeitsangebot und -nachfrage neue intermediäre Akteure auf (Dolata 2020, S. 186). Die Plattformökonomie gilt als eine der größten Herausforderungen für die Arbeitsregulierung in den entwickelten politischen Ökonomien. Demnach könnte Plattformarbeit eine neue Ära der Arbeitsorganisation und -regulierung einläuten, die grundlegend mit den traditionellen Arbeits- und Beschäftigungsformen und ihrer betrieblichen Organisation bricht.

Über Plattformen ausgeübte Arbeit - nachfolgend Plattformarbeit genannt - kann als „logged labour“ (Huws 2016) verstanden werden und zeichnet sich dadurch aus, dass sie quantifiziert und standardisiert ist sowie stark technologisch überwacht und kontrolliert werden kann. Ihre Sprengkraft für die Arbeitsregulierung entwickelt sie dadurch, dass hier die Beschäftigung nicht in Form arbeitsvertraglich geregelter, abhängiger Beschäftigung stattfindet, sondern in Selbstständigkeit transformiert wird. Dies begünstigt ein weltweites Offshoring von Aktivitäten, senkt die Markteintrittsbarrieren und schafft neue Konkurrenz zwischen Arbeitskräften. Gefördert wird diese Vermarktlichung der Arbeit beispielsweise durch Reputations- und Bewertungsverfahren (Drahokoupil und Fabo 2016) sowie durch auf den Plattformen einsehbaren Rankings der dort Tätigen. Als eine Folge der Digitalisierung von Arbeit wird somit die (weitere) Ausdifferenzierung von Beschäftigungsformen jenseits des Normalarbeitsverhältnisses erwartet (Börner, Kehl und Nierling 2017, S. 45-48). Vielfach handelt es sich dabei um flexibilisierte Erwerbsformen, die zum Beispiel nur zeitlich befristet bestehen oder die nur noch durch lose Koppelungen an Unternehmen gekennzeichnet sind. Zu letzteren gehören die neuen (Solo-)Selbstständigkeiten und freiberuflichen Tätigkeiten, die in 
Bereichen digitaler Arbeit auch als Freelancing bezeichnet werden (Kaiser, Süß und Josephs 2012).

Die Vermittlung von Arbeit an Selbstständige anstelle von angestellten Arbeitnehmer*innen wird mitunter für Plattformen als ökonomisch vorteilhaft angesehen, weil es hierdurch auf der Seite der Plattformarbeiter*innen keine betrieblichen Mitbestimmungsrechte oder auch Informationspflichten gibt. Dies, in Kombination mit den ebenfalls ausbleibenden Versorge- und Absicherungsmechanismen beispielsweise in Bezug auf Verdienstausfälle im Krankheitsfall oder bei ausbleibenden Bezahlungen für bereits erbrachte Arbeitstätigkeiten, führt dazu, dass die Bedingungen, unter denen Plattformarbeit geleistet wird, kritisiert (vgl. z.B. Pongratz und Bormann 2017, S. 171; Klebe 2017) oder sogar in die Nähe prekärer Beschäftigung gerückt werden (Lorig 2015; Strube 2015).

Für gewerkschaftliche und betriebliche Interessenvertretungen stellen sich damit zwei grundlegende Herausforderungen. Die erste Herausforderung betrifft die arbeits- und sozialrechtliche Absicherung der Beschäftigten gerade im Bereich des Crowdworking ${ }^{1}$, also der digital zu erledigenden Plattformarbeit, die nach bisherigem deutschen Recht als Selbstständige betrachtet werden und daher weder die arbeitsrechtlichen noch die sozialrechtlichen Ansprüche von Arbeitnehmern geltend machen können, auch wenn sie sich faktisch in einer abhängigen Selbstständigkeit befinden sollten (vgl. Däubler 2015). In dieser Situation fordern die Gewerkschaften eine Ausweitung des Arbeitnehmerbegriffs, so dass davon auch SoloSelbstständige erfasst werden, die auf Plattformen arbeiten (Greef und Schroeder 2017). Gewerkschaften gehen dieses Problem rechtlicher Institutionen also mit Mitteln des politischen Lobbying an.

Noch vitaler für die Gewerkschaften ist hingegen die zweite Herausforderung, nämlich die Vertretung der arbeitsbezogenen Interessen von Plattformarbeiter"innen und ihre Organisierung als Mitglieder in den Gewerkschaften. Beide Aspekte hängen eng zusammen, denn nur als wirksame Interessenvertretungen können sich die Gewerkschaften bei den Plattformarbeiter*innen profilieren, und gleichzeitig können sie diese nur wirkungsvoll vertreten, wenn sie sich dabei auch auf Organisationsmacht stützen können. Die Bedingungen dafür wiederum sind bei der Plattformarbeit problematisch (dazu auch Benner 2014a; Kramer 2018). Denn erstens sind Plattformen keine echten Arbeitgeber oder können darauf verweisen, dass sie nur Arbeit vermitteln; zweitens fördert Plattformarbeit individualisierte

1 Für einen Überblick über die verschiedenen Begrifflichkeiten - Plattformarbeit, Crowdwork, Gigwork - vgl. Abschnitt 2. 
Arbeitsbeziehungen; und drittens weist Plattformarbeit keine betrieblichen Strukturen auf. Deshalb gibt es weder Kopräsenz und Austausch zwischen den Beschäftigten über mögliche Arbeitsprobleme, noch können die Beschäftigten, zumindest im Bereich des Crowdworking - bei den ortsgebundenen Gigworker"innen kann sich dies anders darstellen -, nach aktueller Rechtslage durch Betriebsräte vertreten werden, sei es, weil sie SoloSelbstständige sind, sei es, weil sie keinen Betrieb bilden. Damit entfallen die sozialen Kontexte, die üblicherweise Gewerkschaftsmitgliedschaft begünstigen. Erschwerend kommt hinzu, dass Plattformen häufig transnationalen Charakter haben und sie ihre Crowd weltweit rekrutieren.

Dennoch lassen sich im Bereich der Plattformarbeit erste Initiativen feststellen, die die Interessenorganisation und -vertretung der selbstständigen Plattformarbeiter*innen, von Crowd- und von Gigworker*innen zum Ziel haben. Zu unterscheiden ist dabei zwischen gewerkschaftlich organisierten Initiativen auf der einen und von den Beschäftigten ausgehenden (,grass roots ${ }^{6}$ ) Initiativen auf der anderen Seite. Einige Beispiele für derartige Initiativen sollen im Folgenden eingehender beleuchtet werden: $\mathrm{Zu}$ nächst wird eine Initiative zur Gründung von Betriebsräten bei Essenslieferdiensten betrachtet, die sich noch am ehesten auf Mitbestimmungsrechte berufen konnte. Anschließend daran werden die Initiative Fair Crowd Work der IG Metall (in Kooperation mit anderen Gewerkschaften) sowie die Beratung für Selbstständige der Dienstleistungsgewerkschaft ver.di als gewerkschaftliche Kampagnen im Bereich der selbstständigen Plattformarbeit betrachtet. Abschließend soll anhand von Beispielen und Initiativen aus dem Bereich digitaler Selbstständigkeit wie dem Bloggen diskutiert werden, wie es bei individualisierter und formal selbstständiger Arbeit überhaupt zu einem Austausch über Arbeitsbedingungen und geteilte Interessenlagen in Bezug auf bezahlte Arbeit kommen kann.

Wir sprechen dabei von Interessenartikulation in einem doppelten Sinne. Zum einen geht es uns um die Frage, wie und unter welchen Bedingungen kollektive Interessen in einem Rahmen atomisierter oder individualisierter Arbeitsprozesse und Beschäftigungsbedingungen überhaupt ausgedrückt werden und welche Interessen dies sind. Hier hat Artikulation die Bedeutung von Formung oder Konstruktion von Interessen. Zum anderen sollen die Aktivitäten von Gewerkschaften und anderen Akteuren auf diesem Terrain beleuchtet werden. Untersucht werden somit deren Versuche, solche Konstruktionen zu unterstützen oder überhaupt erst anzustoßen. Ihr Ziel ist es dabei eine Verbindung oder Artikulation herzustellen zwischen der Verbandsebenen und diesen neuen Formen der Beschäftigung mit dem Fernziel, diese kollektiven Interessen auch selber zu organisieren. 
Empirische Grundlage für diesen Text sind Internetrecherchen, Inhaltsanalysen von Onlinedokumenten, Sekundäranalysen und Experteninterviews, die mit Verantwortlichen der Initiativen geführt wurden. Letztere wurden im Rahmen des EU-Forschungsprojekts „Don't Gig Up“ durchgeführt ${ }^{2}$, weiterhin speist sich die Empirie aus Erkenntnissen einer CAIS-Arbeitsgemeinschaft zum Thema „Was bieten Plattformen der Crowd?"3 sowie dem von der MERCATOR-Stiftung gefördertem Projekt „BlogA - Entgrenzte Arbeit im Netz: Bloggen und Vloggen als neue digitale Arbeitsformen" (Hoose und Rosenbohm 2020).

Mit dem exemplarischen Blick auf die vorgestellten Initiativen wird folgenden Fragen nachgegangen: Wie gehen Beschäftigte mit den oben skizzierten Herausforderungen von Plattformarbeit um? Welche Ansatzpunkte einer aktiven Interessenvertretungspolitik und Organisierung gibt es trotz der ungünstigen Bedingungen? Welche Ansätze eignen sich hier für Interessenorganisationen wie Gewerkschaften? Und welche Initiativen entfalten die Beschäftigten möglicherweise selber?

Der vorliegende Beitrag ist wie folgt gegliedert: Im Anschluss an eine Vorstellung von Plattformarbeit, den wichtigsten diesbezüglich verwendeten Begriffen sowie einer knappen Darstellung empirischer Erkenntnisse hierzu (Abschnitt 2) werden vier Beispiele für Interessenvertretungsansätze bei Plattformarbeit vorgestellt. Dies sind die Initiativen ,Liefern am Limit im Bereich Gigwork (Abschnitt 3), ,Fair Crowd Work' im Bereich Crowdwork (Abschnitt 4) sowie die Selbstständigenberatung von ver.di (Abschnitt 5). Zusätzlich werden erste Ansätze für die Organisierung von Interessenartikulation von Blogger"innen und Vlogger"innen vorgestellt (Abschnitt 6), bevor mit einem zusammenfassenden Fazit geendet wird.

\section{Digital ermöglichte Plattformarbeit}

Bevor jedoch auf die Beispiele im Einzelnen eingegangen wird, soll zunächst dem Begriff Plattformarbeit Aufmerksamkeit geschenkt werden:

2 Insgesamt wurden hier fünf Interviews geführt: Jeweils zwei mit ,Liefern am Limit‘ und der Selbstständigenberatung von ver.di sowie eines mit ,Fair Crowd Work'.

3 Die vom Center for Advanced Internet Studies (CAIS) geförderte Arbeitsgemeinschaft wurde gebildet durch Fabian Hoose sowie Paul-Fiete Kramer (Ruhr-Universität Bochum) und fragte danach, wie Plattformbetreiber den sozial konstituierten Markt für Crowdwork in ihrem Sinne zu beeinflussen versuchen, in dem sie ein bestimmtes Bild von Plattformarbeit zeichnen. Hierzu wurden die Internetauftritte von 89 deutschsprachigen Crowdwork-Anbietern inhaltsanalytisch untersucht. 
Ganz allgemein wird darunter all jene Arbeit verstanden, die gegen Bezahlung ${ }^{4}$ auf Plattformen oder durch Plattformen vermittelt geleistet wird (siehe zur Definition des Begriffs auch Hoose und Rosenbohm 2020). Es gibt dabei Formen von Plattformarbeit, bei denen Tätigkeiten rein onlinebasiert erledigt werden (sogenanntes Cloudwork; Kirchner 2019, S. 4). Zudem existieren Formen von Plattformarbeit, deren Erbringung ortsgebunden erfolgt (sogenanntes Gigwork; Schmidt 2016, S. 5). Prominente Beispiele hierfür ist die durch Plattformen wie Uber (Vermittlung von Personenbeförderung) oder Lieferando (Lieferung von online bestelltem Essen) vermittelte Arbeit. Sowohl hinsichtlich Cloudwork- wie auch GigworkPlattformen kann darüber hinaus als weiteres Unterscheidungskriterium herangezogen werden, ob die Vermittlung personengebunden (wie z.B. auf MyHammer, einem Portal für Handwerksdienstleistungen) oder an eine offene Gruppe (die Crowd) gerichtet erfolgt. Letzteres wird als Crowdwork bezeichnet (Schmidt 2016, S. 5; Hensel 2016, S. 169).

Weil bei der Vermittlung von Arbeitstätigkeiten über Online-Plattformen auch bislang innerhalb von Unternehmen vorgenommene Tätigkeiten an eine Crowd ausgelagert bzw. outgesourct werden, hat sich für diese Art der Arbeitsvermittlung der Begriff „Crowdsourcing“ (Howe 2006) etabliert. Allerdings ist mit näherem Blick auf verschiedene Plattformen mittlerweile eine enorme Bandbreite unterschiedlicher Geschäftsmodelle festzustellen, die sich mehr oder weniger direkt auf die Strukturierung von Arbeit durch die Plattformen auswirken. Obwohl es bei diesen Anbietern jeweils eine Ähnlichkeit des Prinzips gibt, über eine Online-Plattform Anbieter*innen und Nachfrager*innen von Arbeit zusammenzubringen, unterscheiden sich die einzelnen Plattformen zum Teil recht deutlich voneinander.

Die Rolle der Plattformen geht dabei über diejenige von reinen Arbeitsvermittlern weit hinaus: Die Unternehmen der Plattformökonomie organisieren den Austausch unterschiedlichster Produkte und Dienstleistungen. Schmidt (2016, S. 6) unterscheidet hier beispielsweise zwischen Plattformen, über die der Austausch verschiedenster Güter und Produkte (z.B. Amazon), von Geld (in Form von Crowdfunding-Kampagnen wie z.B. auf

4 Selbstverständlich gibt es auch unbezahlte bzw. nicht entlohnte Arbeit und gerade im Bereich der Plattformökonomie kommt unentgeltlich geleisteten Tätigkeiten, z.B. in Form von Konsumententätigkeiten (Bauer und Gegenhuber 2015) eine besondere Bedeutung zu (ein Beispiel sind Produktbewertungen in diversen OnlineShops). Zudem sind die Grenzen zwischen unbezahlten, als Hobby oder Freizeitaktivität über Plattformen geleisteten Tätigkeiten, und solchen Handlungen, die als Teil eines Erwerbseinkommens getätigt werden, fließend. 
Kickstarter), von Kommunikation (z.B. auf der Social-Media-Plattform Facebook), von Unterhaltungsangeboten (wie z.B. auf Videoportalen wie YouTube), von Informationen (z.B. Hotelbewertungen wie auf Holidaycheck) oder die Vermittlung bezahlter Dienstleistungen (z.B. auf Clickworker.de) organisiert wird. Charakteristisch für die Unternehmen der Plattformökonomie (also: für die Plattformunternehmen bzw. Plattformen) ist, dass sie eine (digitale) Infrastruktur errichten, über die ein (marktlicher) Austausch von Angebot und Nachfrage ermöglicht wird. Die Plattformen treten dabei als neue Intermediäre zwischen den Marktteilnehmer*innen auf und strukturieren den Austausch, ermöglichen eine Delegation verschiedenster Aufgaben auf unterschiedlichste Akteure und erlauben zudem deren dezentrale und mobile Bearbeitung (Kirchner und Beyer 2016, S. 327-329).

Ein Teil der Plattformen kann als Arbeitsplattformen bezeichnet werden (Pesole, Urzí Brancati, Fernández-Macías, Biagi und González Vázquez 2018, S. 7): Einerseits, weil sie Arbeit für bezahlte Dienstleistungen vermitteln, andererseits, weil sie Arbeitenden (zumeist Selbstständigen) einen direkten Marktzugang ermöglichen, bei dem diese ihre Arbeitsleistung direkt über die Plattform an Kunden verkaufen können. Die Plattformen nutzen die ,Crowds', also jeweils eine Vielzahl von potenziellen Auftragnehmern, zur Etablierung ihrer Geschäftsmodelle und sind gleichsam auch die Initiatoren dieser Ansammlungen von Arbeitssuchenden. Durch Plattformregeln „orchestrieren“ (Dickel und Thiem 2018, S. 252) die neuen Intermediäre die Bezahlung, Kontrolle und Zugänge zu Arbeitsmöglichkeiten. Damit werden von den Plattformen die Arbeitsbedingungen (vor)strukturiert. Charakteristisch für Plattformarbeit ist, dass das Verhältnis von Plattformen zu den Plattformarbeitenden als "lose Kopplung“ (Kirchner und Beyer 2016, S. 327) beschrieben werden kann - was zugleich ein zentraler Unterschied zu betrieblich organisierter Arbeit ist, die eine örtliche Bindung von Arbeitskraft und Unternehmen bedeutet (Gerber und Krzywdzinski 2019, S. 25).

„Den typischen Crowdworker gibt es nicht“ (Altenried 2017, S. 184), sondern die Plattformarbeiter*innen unterscheiden sich hinsichtlich ihrer soziodemografischen Merkmale, ihrer Qualifikationen etc. mitunter sehr stark - selbst dann, wenn sie die gleichen Tätigkeiten auf Plattformen übernehmen. Daraus scheinen mitunter auch unterschiedlich gelagerte Interessen zu erwachsen: So können Plattformarbeitende aus verschiedenen Ländern unterschiedliche Ansprüche an eine ,gute' Bezahlung haben; Studierende haben möglicherweise geringere Ansprüche an die soziale Absicherung durch Plattformarbeit als Alleinerziehende und hochqualifizierte Selbstständige mit weiteren Einkommensquellen sehen sich mitunter we- 
niger von Plattformregeln und Bewertungen durch Auftraggeber abhängig an als Personen ohne formale Bildungsabschüsse, deren Plattformranking mitunter ihr einziger Ausweis über das individuelle Arbeitsvermögen ist.

Leimeister, Durward und Zogaj (2016) konnten zeigen, dass die meist recht jungen Crowdworker*innen (36 Jahre im Durchschnitt) häufig auf mehreren Plattformen zugleich aktiv sind und für sie relativ geringe Verdienstmöglichkeiten bestehen. $\mathrm{Zu}$ ähnlichen Befunden kommen Bertschek, Ohnemus und Viete (2015, S. 9), wonach 69\% der Crowdworker*innen unter 19 Euro pro Woche verdienen. Die Mehrheit (65\%) erhält zudem für einen erledigten Arbeitsauftrag durchschnittlich weniger als zwei Euro. Auffällig ist das durchaus hohe Bildungsniveau der Befragten (Leimeister et al. 2016, S. 73). Allerdings ist Crowdwork für die Mehrzahl der so Beschäftigten auch nicht die hauptsächliche Einkommensquelle. Zumeist wird Crowdwork genutzt, um einen (schnellen) Nebenverdienst zu ermöglichen, ohne dass zwingend spezielle Kenntnisse oder berufsfachliche Qualifikationen notwendig sind.

Obgleich die derzeitige empirische Bedeutung plattformvermittelter Arbeit noch als begrenzt angesehen werden kann (Erdsiek, Ohnemus, Viete 2018, S. 7-9), haben Analysen von Crowd- und Gigwork bei der Untersuchung des digitalisierungsgetriebenen Wandels von Arbeit mittlerweile einen festen Platz eingenommen. Dies liegt einerseits darin begründet, dass zwar die Zahl der hauptberuflich über Plattformen Arbeitenden (in Deutschland) gering ist, Crowdwork als zusätzlicher Nebenverdienst jedoch durchaus verbreitet ist. So geht Serfling (2018, S. 13) nach einer Befragung von 376.750 Internetnutzer*innen von einem Anteil von bis zu 4,8\% aktiver Crowdworker*innen unter der Wahlbevölkerung Deutschlands aus. Nach Urzí Brancati, Pesole und Férnandéz-Macías (2020, S. 16) bemisst sich der Anteil der Plattformarbeitenden auf bis zu 5,7\% an der Bevölkerung im Erwerbsalter in Deutschland, die dadurch den hauptsächlichen oder zumindest einen relevanten Anteil ihrer Erwerbstätigkeit bestreiten ${ }^{5}$. Auch die zum Teil von den Plattformen selbst verbreiteten Nutzerzahlen deuten durchaus auf eine relevante Anzahl aktiver Plattformarbeiter*innen hin: So vermeldet zum Beispiel der Anbieter ,Clickworker', im Jahr 2020 auf über 2,2 Millionen registrierte Plattformarbeitende zurückgreifen zu können. ${ }^{6}$

5 Das bedeutet, dass die Plattformarbeitenden mindestens $25 \%$ ihres Einkommens oder aber mindestens 10 Stunden pro Woche über Plattformen arbeiten (Urzí Brancati, Pesole, Férnandéz-Macías 2020, 15-16).

6 Vgl. https:/www.clickworker.de/ueber-uns/unsere-crowd-die-clickworker/ (zuletzt abgerufen am 13.08.2020). 
Doch nicht nur die Zahl der Crowdworker*innen ist ein Indiz für die Bedeutung plattformvermittelter Arbeit: Hoffmann und Suchy (2016, S.27) verweisen darauf, dass der Blick auf (wenige) hauptberufliche Crowdworker“innen „völlig zu Unrecht“ dazu führe, diese Form der Arbeitsorganisation als unbedeutend einzustufen. Seine Relevanz erhielte das Thema Crowdsourcing stattdessen vielmehr dadurch, dass immer mehr Unternehmen austesten würden, inwieweit sie zumindest Teile ihrer Wertschöpfung über die Auslagerung auf Crowds restrukturieren können. Damit vermögen es plattformvermittelte Erwerbsformen, traditionelle und regulierte Beschäftigung (in Unternehmen) unter Druck zu setzen (vgl. hierzu Greef und Schroeder 2017; Altenried 2017). Gerade aus Reihen der (DGB-)Gewerkschaften gibt es daher Forderungen danach, „einen Wettbewerbsrahmen für Plattformen und einen Gestaltungsrahmen für Crowdwork" (Hoffmann und Suchy 2016, S. 26) zu schaffen.

Grundsätzlich kann zwischen zwei Tätigkeitsgruppen unterschieden werden, die über Plattformen vermittelt werden und die sich auf die Ausgestaltung der Plattform und der dortigen Arbeitsbedingungen auswirkt: $\mathrm{Zu}$ finden sind „einerseits standardisierte, in kleine Arbeitspakete heruntergebrochene Routine- und Unterstützungsaufgaben wie Textproduktion, Datenkategorisierung oder Umfragen (sogenannte Mikroaufgaben); andererseits kreative Lösungen, die ebenfalls relativ standardisiert sein können - wie teilweise im Design-Bereich - oder aber hochgradig spezialisiert und wissensintensiv sind wie im Bereich der Programmierung oder Innovation (sogenannte Makroaufgaben)“ (Gerber und Krzywdzinski 2017, S. 6; vgl. auch Schönefeld und Hensel 2019, S. 13-14).

Gerade für die Gruppe derjenigen, bei denen Plattformarbeit eine wichtige Einnahmequelle zum Lebensunterhalt ist, können sich die oftmals einseitig auf die Belange der auftraggebenden Unternehmen abzielenden Plattformregeln als problematisch erweisen. Den Auftraggeber“innen liefern die Plattformen häufig viele Informationen über die potentiellen Bearbeiter*innen von Tätigkeiten, zum Beispiel indem diese mittels verschiedener Bewertungssysteme in einem Ranking geführt werden. Im Gegenzug können sich die Crowdworker*innen ihrerseits jedoch nur in engen Grenzen ein Bild über die Auftraggeber*innen machen. Dies führt insbesondere dann zu Problemen, wenn Zahlungen für erledigte Tätigkeiten zurückgehalten werden (z.B., weil die Vergabe über Wettbewerbe organisiert wird, bei denen nur ausgewählte Arbeitsergebnisse bezahlt werden). Wie im Folgenden exemplarisch anhand mehrerer Beispiele gezeigt wird, haben sich unter anderem aus derartigen Beweggründen verschiedene Initiativen entwickelt, welche diese Machtungleichgewichte abzubauen versuchen. 


\section{Beispiel 1: Organisation von Interessen bei lokalem Gigwork - „Liefern am} Limit"

Das erste der hier angeführten Beispiele betrifft Betriebsratsgründungen im Bereich der Essenslieferdienste. In Deutschland gibt es inzwischen ein breites Netz solcher Dienstleistungen, die von multinationalen Unternehmen wie Foodora, Lieferando oder Deliveroo in städtischen Gebieten angeboten werden bzw. wurden. ${ }^{7}$ Initiativen zu Betriebsratsgründungen finden sich in mehreren Unternehmen der Branche; im Zentrum der Betrachtung steht hier die Entwicklung bei Deliveroo, einem international operierenden Unternehmen mit Sitz in London (UK), welches bis August 2019 auch in Deutschland tätig war. Hier haben die Initiator*innen der Betriebsratsgründung eine überaus erfolgreiche Medienkampagne mit dem Titel „Liefern am Limit“ gestartet, in der sie die Arbeitsbedingungen bei den Zustelldiensten skandalisiert und einer breiten Öffentlichkeit bekannt gemacht haben.

Online-Essenslieferdienste beliefern Kund*innen mit Getränken und Speisen, die in Partner-Restaurants zubereitet werden. Die Arbeitsorganisation ist dabei „beinahe vollständig digitalisiert“ (Palmer 2017, S. 29). Die Arbeitsaufträge an die auch ,Rider ${ }^{`}$ genannten Fahrer*innen werden mittels Algorithmus verteilt: Eingehende Aufträge werden angenommen; dann ortet der Algorithmus den bzw. die nächste verfügbare Fahrer*in und sendet ihm oder ihr den Auftrag. Bei Deliveroo war zum Ausgangspunkt der Initiative das Gros der Beschäftigten befristet angestellt, mit Arbeitsverträgen von sechs Monaten. Dazu gab es - wenige - Freelancer*innen, die als Solo-Selbstständige auftragsweise arbeiteten. Die Bezahlung der Freelancer*innen erfolgte pro Fahrt, die Angestellten erhielten einen festen Stundensatz. Die Rider selber wurden mit Rucksack, Regenjacke, Regenhose und T-Shirts ausgestattet, den Rest der Ausrüstung mussten sie selbst stellen. Das betraf insbesondere ein zur Ausübung der Tätigkeiten unabdingliches Smartphone samt Datentarif sowie ein Fahrrad. Bei Deliveroo gab es zudem ein weiteres Problem, das in den Deutungen der Beschäftigten als ungerecht empfunden wurde und schließlich zur Gründung eines Betriebsrats führte: Die Lohnabrechnungen, die bei vielen Be-

7 Derzeit hat sich der Markt hier konsolidiert. Nach der Übernahme von Foodora durch das niederländische Unternehmen Takeaway.com (u.a. mit der Marke Lieferando) Ende des Jahres 2018 und dem Rückzug aus dem Deutschlandgeschäft des britischen Unternehmens Deliveroo im August 2019 wird die Branche faktisch von einem Unternehmen dominiert (Heiland und Brinkmann 2020, S. 123). 
schäftigten falsch oder unvollständig waren, und dies zumeist zuungunsten der Beschäftigten.

„Als ich anfing, begannen auch schon Probleme. Ich habe im ersten Monat keine Lohnabrechnung bekommen, das wurde nicht richtig an das Abrechnungsbüro gemeldet. Ich bin dann auch ins Office gegangen und habe gefragt, ob ich nicht wenigstens einen Abschlag bekommen könnte, aber das hat man verweigert und gesagt, es würde dann alles mit der nächsten Abrechnung nachgezahlt. Es gab da keinen Spielraum. "(Expertin Deliveroo)

Ein Zentrum der Aktivitäten entwickelte sich in der Stadt Köln. Hier war im Sommer 2017 bereits ein Betriebsrat bei einem damaligen Konkurrenzunternehmen, Foodora, gegründet worden. Von dort wechselten einige Aktive zu Deliveroo und waren zunächst verwundert, dort ähnliche Probleme wie bei ihrem alten Arbeitgeber zu finden:

„Es waren dann drei Leute, die das vorangetrieben haben und die einen Betriebsrat gründen wollten. Sie sind dann zur NGG gegangen und haben sich beraten lassen, was sie dafür tun müssen. "(Expertin Deliveroo)

Dieses Vorhaben sollte sich als steiniger Prozess erweisen. Dies zeigte sich an zwei Entscheidungen des Unternehmens nach der Bekanntgabe der Initiator*innen, dass sie einen Betriebsrat gründen wollen. Die erste betraf die Social-Media-Plattform des Unternehmens. Diese Plattform ermöglichte die Kommunikation sowohl mit den anderen Beschäftigten am Standort Köln als auch, auf einem zweiten Kanal, mit den Beschäftigten in ganz Deutschland. Die Initiator*innen sendeten im September 2017 über die deutschlandweite Vernetzung die Information, einen Wahlvorstand für einen Betriebsrat in Köln gründen zu wollen. Weniger als eine Stunde später hat das Unternehmen die Plattform abgeschaltet. Kommunikation zwischen den Beschäftigten über Unternehmenskanäle ist seitdem nur noch über die Zentrale des Unternehmens in Berlin möglich.

Die zweite Entscheidung betraf die Initiator*innen selbst. Als sie dem Unternehmen offiziell die Gründung eines Wahlvorstands bekanntgaben, wurde der Hauptinitiator, der spätere Betriebsratsvorsitzende, in der Organisation degradiert. Er war zwar auch Rider, hatte aber zugleich als Mentor die Fahrer*innen in den Büroräumen des Unternehmens, dem „Office", beraten und betreut. Nach der Ankündigung wurde ihm verboten das Office weiter zu betreten; er durfte nur noch Aufträge ausliefern. Damit hat das Unternehmen eine regulatorische Schwäche des deutschen Betriebsverfassungsgesetzes ausgenutzt. Vom Gesetz werden zwar Wahlvorstände vor Entlassungen oder andere Diskriminierungen geschützt, während der Vorlaufzeit zur Wahl gibt es aber keinen rechtlichen Schutz. 
Nachdem der Wahlvorstand trotz dieser Widrigkeiten gegründet wurde, versuchte das Unternehmen das Heft des Handelns in die Hand zu nehmen und Zeit und Ort der Wahl zu bestimmen, um so den Wahlprozess weiter zu behindern. Dennoch fand die Wahl fand schließlich im Office statt; rund 30 von etwa 200 Beschäftigten am Standort haben an der Wahl teilgenommen. Alle Teilnehmer*innen waren Fahrer*innen.

Für die Informationen vor und nach der Wahl mussten alternative Kommunikationskanäle nutzbar gemacht werden, denn die Kommunikation über die Social-Media-Plattformen des Unternehmens war gesperrt. Alternativ wurden zwei WhatsApp-Gruppen unter den Fahrer"innen eingerichtet. Eine davon dient nur dazu, sich für den Feierabend zu verabreden. Die andere ist das Forum für Kritik. Dank der Kommunikation dieser Gruppe wurde das Wissen um die vielen Abrechnungsfehler verbreitet; hier erfuhren die Fahrer*innen auch von Klageverfahren jener Rider, die von der Gewerkschaft Nahrung-Genuss-Gaststätten (NGG) unterstützt werden.

Unmittelbar nach der Wahl begann das Unternehmen, die Angestellten durch Freelancer"innen zu ersetzen. Auslaufende befristete Verträge wurden nicht verlängert, dafür wurden Aufträge vermehrt an Freelancer"innen vergeben. Im November 2017 gab es etwa 140 angestellte Rider, im Februar nur noch rund 20. „Innerhalb von drei Monaten ließ das Unternehmen mehr als die Hälfte der 100 befristeten Arbeitsverträge am Standort Köln auslaufen“ (Zander 2018, S. 17).

Der Betriebsrat hat zunächst versucht, vom Unternehmen Informationen und Daten zu den Beschäftigungsverhältnissen und zur Bezahlung zu bekommen. Er wollte auf diese Weise seine Mitbestimmungsmöglichkeiten bei der Gestaltung der Beschäftigungsverhältnisse ausloten. Im Zuge der Betriebsratswahl und den zahlreichen Behinderungen entstand zudem bei den Initiator*innen die Idee, mit den Problemen an die Öffentlichkeit zu gehen.

„Wir haben uns überlegt, wie Öffentlichkeitsarbeit aussehen könnte. Wir haben dazu auch Informationen bei der Gewerkschaft NGG eingeholt. Wir wollten eine Öffentlichkeit herstellen. Als erstes haben wir ein Plakat entwickelt mit dem Spruch „Liefern am Limit“." (Expertin Deliveroo)

Mit dem Plakat wurde auf einem Platz in der Kölner Innenstadt eine Demonstration organisiert. Die Aktion war sehr werbewirksam; sowohl Zeitungen als auch Radio und das öffentlich-rechtliche Fernsehen waren vor Ort. Auch Fahrer*innen des Konkurrenten Foodora - die Fahrer*innen waren in freundschaftlicher Konkurrenz verbunden - kamen hinzu und gesellten sich zu den Demonstrierenden. Darauf entfaltete sich eine Spirale 
wachsenden Interesses, die die Initiator*innen bis zum Bundesarbeitsminister führte.

„Wir haben der Presse Interviews gegeben, und dann wurde das Interesse immer größer. Der Betriebsratsvorsitzende war später bei einer Polit-Talk-Sendung im Fernsehen, dort war auch der Bundesarbeitsminister, und hinterher haben sich die beiden bei einem Bier intensiv unterhalten. So haben wir auch Kontakt zum Arbeitsminister bekommen. Der hat uns dann auch einmal in Köln besucht und rund eineinhalb Stunden sehr intensiv mit uns gesprochen." (Expertin Deliveroo)

Die Initiator*innen richteten zudem eine Seite auf Facebook zu „Liefern am Limit" ein, die sie bis heute aktualisieren. Das Ziel ist öffentlich zu machen, was bei den Essenslieferanten passiert. Auch Interviewanfragen und Einladungen zu Tagungen und Talkshows zeugen von einem ungebrochenen Interesse an dem Thema.

„Liefern am Limit“ war eine Initiative der Fahrer"innen. Aber die Gewerkschaft NGG hat in dem Prozess eine wichtige unterstützende Rolle gespielt. Sie hat die Initiator*innen bei der Betriebsratsgründung beraten, und sie hat mit an dem Konzept für die Öffentlichkeitsarbeit gefeilt, und auch heute finden in den Büros der Gewerkschaft Strategiesitzungen statt, wie man die Kampagne fortsetzen sollte. Zudem berät die Gewerkschaft bei der Pressearbeit, die immer aufwändiger geworden ist. Ein Mitglied des ursprünglichen Betriebsrats von Deliveroo ist zur Gewerkschaft gewechselt und ist dort nun Projektsekretär für die Lieferdienste. Das Fernziel der Gewerkschaft lautet, Tarifverträge mit den Lieferdiensten abzuschließen.

„Das ist sehr gut; nun gibt es dort jemanden, der die Arbeit koordiniert und der über Ressourcen verfügt. Man weiß an wen man sich wenden kann, und es gibt mehr Unterstützung. Das Ziel der Gewerkschaft ist klar: Die wollen bei den Lieferdiensten Tarifverträge durchsetzen. Im Zentrum steht da jetzt Foodora." (Expertin Deliveroo)

Zum Zeitpunkt der hier zitierten Interviews im Jahr 2019 bestand der Betriebsrat am Kölner Standort von Deliveroo noch aus einer Person. Bereits vier der fünf Betriebsräte waren nicht mehr da, weil ihre Arbeitsverträge nicht verlängert wurden. Drei von ihnen haben gegen diese Entscheidung geklagt und einen gerichtlichen Vergleich angenommen. Der Betriebsratsvorsitzende war zunächst noch im Amt, er hatte den Vergleich nicht akzeptiert. Das Gericht hatte entscheiden, dass er nicht sachgerecht befristet war und wiedereingestellt werden musste. Das Unternehmen rief daraufhin die höhere gerichtliche Instanz an. 
Auch in Münster gab es eine Betriebsratsinitiative bei Deliveroo; dort hatte das Unternehmen den Betriebsrat nicht anerkannt und argumentiert, dass es dort kein Office und damit keinen Betrieb habe. Die Fahrer*innen dort würden deshalb zu Köln gehören und müssten hier ihren Betriebsrat wählen.

Die Konflikte um Betriebsratsgründungen zwischen der Initiative Liefern am Limit und Deliveroo endeten damit, dass das Unternehmen Mitte 2019 recht kurzfristig seinen Betrieb in Deutschland einstellte: Die Initiative bewertete den Rückzug mit der Feststellung: „das Modell der (schein-)selbstständigen Fahrradkurier ist gescheitert" (Liefern am Limit, Facebook-Eintrag vom 13.08.2019). Die Initiative besteht jedoch weiterhin und hat seine Aktivitäten mit Hilfe der Gewerkschaft NGG auch auf weitere Lieferdienste ausgeweitet.

Beim Lieferdienst Foodora, einem direkten Konkurrenten von Deliveroo der inzwischen von Takeaway.com mit der Plattform Lieferando, dem letzten verbliebenden Mitbewerber in diesem Feld, übernommen wurde, war die Betriebsratsgründung zunächst weniger umkämpft. Auch hier konnte am Standort Köln ein Betriebsratsgremium gewählt werden. Die strukturellen Bedingungen dafür waren besser, weil dort die befristeten Arbeitsverträge eine Laufzeit von einem Jahr hatten und weil das Unternehmen nicht mit Freelancer*innen arbeitete, also nicht befristet Beschäftigte durch Freelancer*innen ersetzt hatte. Probleme aus den Befristungen ergaben sich aber trotzdem.

„Bei Foodora gibt es auch einen neuen Betriebsrat in Köln, die sind so rund 250 Leute. Der will sich jetzt neu wählen lassen, weil es einige Umstellungen gegeben hat, dort wurde der stellvertretende Vorsitzende nicht verlängert und musste ausscheiden." (Expertin Deliveroo)

Die Initiative Liefern am Limit engagiert sich somit auch nach dem Marktaustritt von Foodora und Deliveroo weiter im Bereich der Essenslieferdienste. Wurde nach dem Rückzug von Deliveroo zunächst noch auf bessere Arbeitsbedingungen bei Lieferando gehofft, bestehen inzwischen alte und neue Konfliktlinien sowohl hinsichtlich Betriebsratsgründungen und der Befristung von Arbeitsverträgen, aber auch bezogen auf die Aushandlung von Tarifverträgen sowie die Frage nach angemessenen und sicheren Arbeitsbedingungen (insbesondere bei schlechtem Wetter sowie unter den Bedingungen der Corona-Virus-Pandemie; vgl. Altenried, Niebler und Wallis 2020). Die Prozesse der Betriebsratsgründungen zeigen, wie solche Interessen geweckt und politisiert werden können. Das Beispiel ,Liefern am Limit ${ }^{6}$ deutet zudem auch an, wie Gewerkschaften Interessenvertretungsansprüche von Plattformarbeitenden aufnehmen und sich unterstüt- 
zend einbringen können - auch in einem Umfeld, welches nicht unbedingt als Kernzielgruppe bisheriger gewerkschaftlicher Aktivität anzusehen ist.

\section{Beispiel 2: Ansprache von Plattformarbeiter"innen - Fair Crowd Work}

Neben dem Bereich des lokalen Gigworks hat in der Forschung zur Arbeit in der Plattformökonomie auch die digitale Verteilung und Bearbeitung von Kleinstaufgaben, das sogenannte „Microworking“ (Schönefeld und Hensel 2019, S. 17) einige Aufmerksamkeit erfahren. Während im Fall von Essenslieferdiensten nur wenige Plattformbetreiber vorhanden sind und die Plattformarbeiter*innen dort häufig, wenn auch befristet, arbeitsvertraglich gebunden sind, stellt sich die Situation bei der digital und ortsungebunden ausgeübten Plattformarbeit anders dar: Hier existiert eine Vielzahl von Plattformen, die sich aber zumindest darin ähneln, dass die dort jeweils tätigen Crowdworker*innen formal selbstständig tätig sind.

In der Diskussion um die Arbeitsbedingungen bei Crowdwork wurde insbesondere kritisiert, dass es hier keine stabilen Einkommen gibt, die Vergütung einzelner Tätigkeiten sehr gering ausfällt und es darüber hinaus keinerlei soziale Absicherung der Crowdworker*innen gibt. Angesichts derartiger Missstände - verbunden mit der Befürchtung, Crowdwork könne das Potential entwickeln, Formen der abhängigen Beschäftigung zu verdrängen - sind auch Gewerkschaften auf das Thema aufmerksam geworden. Die wohl aufwändigste gewerkschaftliche Initiative der Verbreitung von Interessenvertretungsstrukturen in der Plattformökonomie ist die Initiative Fair Crowd Work der IG Metall, welche im Jahr 2015 startete. Kurz zuvor hatte die heutige Zweite Vorsitzende der Gewerkschaft, Christiane Benner, ein Buch mit dem Titel „Crowdwork - Zurück in die Zukunft? Perspektiven digitaler Arbeit“ (Benner 2014b) herausgegeben. Dieses Buch hat die gewerkschaftliche Debatte über Crowdwork eröffnet und geprägt.

In dem Buch benennt Benner drei Gründe, warum Gewerkschaften sich mit Crowdwork beschäftigen sollten: Weil die Arbeit in der Online-Welt Auswirkungen auf die Arbeitsbedingungen aller Beschäftigten haben wird; weil auch Online-Arbeit Arbeit ist, die fair entlohnt und reguliert werden sollte, und weil es gelte, einen sozialen Rückschritt zu verhindern, der die Gesellschaft an den Beginn des Industriezeitalters zurückwerfen könnte. Sie forderte, dass Schutzrechte des Wirtschaftslebens wie Urheberrechte und Allgemeine Geschäftsbedingungen auch für Crowdworker*innen gelten müssen, dass Schutzrechte für Beschäftigte auf Crowdworker"innen Anwendung finden oder dahingehend erweitert werden müssen und dass 
damit digitale Arbeit rechtlich zu gestalten ist, um Mindestbedingungen durchsetzen zu können. Ausdrücklich geht es damit nicht um eine Verhinderung digitaler Arbeit, sondern um ihre soziale Regulierung.

Zeitgleich hat die Gewerkschaft eine erste Version einer Webseite mit dem Titel „Fair Crowd Work" entwickelt und online gestellt. Dabei knüpfte sie an das Browser-Plug-In „Turkopticon“ an, das als Reaktion auf die Plattform „Amazon Mechanical Turk" entwickelt wurde (Silberman und Irani 2016; Ellmer 2016) und mit dem Crowdworker*innen ihre Auftraggeber bewerten können mit dem Fernziel, eine „Workers' Bill of Rights“ zu etablieren. Einer der Vordenker von „Turkopticon“, M. Six Silberman, wechselte wenig später zur IG Metall und übernahm die Betreuung der Plattform ,Fair Crowd Work ${ }^{\varsigma}$.

"The general goal behind the website's design was to create a place for workers to post and read reviews of digital labor platforms. The target users were current or prospective platform workers who would like to make betterinformed decisions about which platforms on which to work. The platform reviews on this original site had two main sections: worker reviews and a 'terms of service check." (Harmon und Silberman 2018, S. 1283)

Im selben Jahr noch wurde das Konzept der Webseite überarbeitet, und ein Jahr später konnte eine zweite Fassung der Seite ins Netz gestellt werden. Die entscheidende Änderung bestand darin, die Methode der Erhebung zu wechseln. In der ersten Fassung wurde die Bewertung direkt von den Besucher*innen abgegeben. Die einzige Voraussetzung dafür war, sich auf der Webseite mit einer E-Mail-Adresse anzumelden; gesicherte Informationen darüber, ob die Bewertenden tatsächlich jemals auf einer Plattform gearbeitet haben, gab es nicht. Dieses Verfahren erschien nicht mehr rechtssicher genug. Der Hintergrund für diese Annahme war die gerichtliche Klage eines Zahnarztes, der gegen eine Bewertung seiner Praxis im Netz geklagt hatte mit dem Argument, dass er den Bewerter, der ihn schlecht bewertet hat, niemals behandelt oder auch nur gesehen hatte. Das Gericht hat in diesem Fall für den Kläger entschieden, das Rating musste gelöscht werden. Diese rechtlichen Probleme sollten vermieden werden.

Deshalb wurde in der zweiten Fassung ein neuer Zugang entwickelt, nämlich eine Befragung der Beschäftigten auf Plattformen. Dazu wurden Plattformen angefragt, sofern sich die Fragebögen dort nicht von außen einstellen ließen, und die meisten der Plattformen - außer als gewerk-

8 Im Internet ist die Plattform unter http://faircrowd.work zu erreichen (Stand November 2020). 
schaftskritisch bekannte Plattformen wie Amazon Mechanical Turk und Uber - haben auch zugesagt. Die IG Metall entschloss sich, den Antwortenden für diese Befragung Geld zu bezahlen. Zum einen sollte damit ein guter Rücklauf sichergestellt, zum anderen sollte dem Charakter des Crowdwork Rechnung getragen werden, mit Klicks Geld zu verdienen. Dabei wurden die Tarife den Gepflogenheiten der Plattformen angepasst; sie lagen zwischen 10 und 14 Euro. Die Fragebögen wurden dann von der IG Metall ausgewertet und auch einem Konsistenztest unterzogen, erst dann wurden die Rankings bearbeitet. Die Zahl der eingegangenen Fragebögen wies eine Spanne von 25 bis 150 pro Plattform auf. Die Befragung war nicht repräsentativ, aber sie lieferte Ergebnisse, die es sonst nicht gab.

„Wir hatten im Jahr 2016 eine große Veranstaltung beim ETUI in Brüssel, dort haben sich die Leute alle beklagt, dass man nichts über die Crowdworker weiß, dass man an die Leute nicht herankäme. Und ich konnte auf der Basis unserer Plattform sagen: Leute, das stimmt so nicht, man kommt an die Leute heran. Es ist nicht einfach, man muss sich etwas einfallen lassen, aber es ist möglich. Und auch die Plattformen machen zum Teil mit. Also, die Botschaft war: Wir sind nicht chancenlos. Und wir haben entschieden, wir wollen jetzt mehr mit den Plattformen reden. "(Experte IG Metall)

Die so nicht erwartete Dialogbereitschaft der Plattformen gab den Anstoß dafür, den Dialog auszuweiten. Die IG Metall entschied sich, neben die Ratingplattform und die Befragung ein zweites Standbein der gewerkschaftlichen Strategie zu stellen, die direkte Einflussnahme auf die Plattformen. Ein wichtiger Anknüpfungspunkt dafür war der Code of Conduct für Plattformen, der im Jahr 2015 von einigen Plattformen in Deutschland vereinbart worden war, um den schlechten öffentlichen Ruf der Plattformen zu verbessern. Der mittlerweile in einer zweiten Version von acht Plattformen unterzeichnete Code of Conduct hat laut Präambel das Ziel, „ergänzend zur Gesetzgebung allgemein gültige Leitlinien für das eigene Handeln im Rahmen von bezahlter Crowdarbeit zu etablieren und so eine Basis für ein vertrauensvolles und faires Miteinander zwischen Plattformbetreibern und Crowdworkern zu schaffen". ${ }^{9}$ Er enthält Punkte wie die Konformität mit rechtlichen Vorgaben, faire - insbesondere transparente Bezahlung, gute Arbeit, respektvoller Umgang, klare Zielvorgaben und vernünftige Zeitplanung, konstruktives Feedback, ein geregelter Bewer-

9 Der Code of Conduct ist einsehbar unter http://www. http://crowdsourcing-code.d e/ (zuletzt abgerufen am 02.05.2019). 
tungsprozess und die Einhaltung des Datenschutzrechts und die Wahrung der Privatsphäre für die Beschäftigten.

Im Vorfeld der Diskussion mit den Plattformen hatte die IG Metall zusammen mit internationalen Gewerkschaften aus Österreich, Schweden, Dänemark sowie den USA und Kanada in Frankfurt einen Workshop abgehalten und im Anschluss die „Frankfurter Erklärung“ veröffentlicht (IG Metall et al. 2016), in der die Gewerkschaften für die Einhaltung des Mindestlohns, Zugang zu sozialer Sicherung, Transparenz und Schlichtungsverfahren plädierten. Mit diesen Punkten ging die IG Metall in Gespräche mit Vertreter*innen der Plattformen, welche den Code of Conduct unterzeichnet hatten. Dabei betonte sie insbesondere die Rolle des Mindestlohns und forderte, dass man auch dann ein überlebensfähiges Einkommen haben müsse, wenn man nur von der Plattformarbeit lebe. Die Plattformen verwiesen auf zwei Probleme: die Schwierigkeit, Arbeitszeit zu messen, und die globale Konkurrenz bei Aufträgen. Außerdem wiesen sie darauf hin, dass der Lohn für Plattformarbeiter"innen zweitrangig sei; es ginge vielmehr um Spaß und Abwechslung. Die IG Metall konnte vereinbaren, zu dieser Frage eine Befragung durchzuführen.

„Dann haben wir gesagt ok, lasst uns doch die Worker befragen, was für sie wichtig ist, und das haben wir dann auch gemacht. Da kam heraus, dass für die Worker faire Bezahlung die mit Abstand wichtigste Größe ist. Da haben die Plattformen gesagt, ok, das überrascht uns, dann müssen wir etwas tun." (Experte IG Metall)

Ein erster Schritt bestand darin, den Code of Conduct zu überarbeiten. Neu wurde das Prinzip eingeführt, dass die Plattformen nach den ortsüblichen Entgelten bezahlen. Das entsprach nicht der Forderung nach Einhaltung des Mindestlohns, wurde von der IG Metall aber als wichtiger erster Schritt gewertet.

„Unsere Forderung nach dem Mindestlohn haben sie nicht direkt aufgenommen und wir kämpfen weiter dafür; aber es war schon mal ein erster Schritt." (Experte IG Metall)

Die überarbeitete Fassung des Code of Conduct wurde 2017 präsentiert und gegenüber der ersten Version von fünf weiteren Plattformen unterschrieben. Ein zweiter wichtiger Schritt erfolgte dann ebenfalls im Jahr 2017, als die IG Metall mit den nun acht Unterzeichner*innen des Codes of Conduct und dem Deutschen Crowdsourcing-Verband (DCV) eine Ombudsstelle (Ombuds Office) eingerichtet hat, die die Umsetzung der Normen des Codes of Conduct sicherstellen und Konflikte zwischen Crowdworker*innen und Plattformen behandeln soll. Die Ombudsstelle ist mit 
fünf Personen besetzt, darunter zwei Vertreter*innen der Plattformen (einer Plattform und des DCV), zwei Vertreter*innen der Beschäftigten (Crowdworker"in und Vertreter"in der Gewerkschaft) und als neutraler Person einem Arbeitsrichter. Seit Eröffnung der Ombudsstelle wurden rund 30 Verfahren behandelt; alle Verfahren konnten mit Einschaltung der Ombudsstelle im Konsens gelöst werden.

Aktuell verfolgt die IG Metall drei weitere Schwerpunkte in ihrer Crowdworking-Initiative. Der erste Schwerpunkt ist die Ausweitung der Unterzeichnerliste des Code of Conduct; die Gewerkschaft spricht mit weiteren Plattformen auch aus dem Bereich des Gigworking und versucht sie zu überzeugen, den Code zu unterschreiben. Der zweite Schwerpunkt ist die Entwicklung einer dritten Fassung der Plattform Fair Crowd Work mit dem Ziel, einen Kriterienkatalog (FAQ) für eine gute Gestaltung der Allgemeinen Geschäftsbedingungen zu erarbeiten und dort einzustellen. Denn mit den AGBs entscheidet sich aus Sicht der Gewerkschaft nicht nur der Status der Beschäftigten, sondern auch die Arbeitsbedingungen mit Blick auf Transparenz und faire Behandlung und Kommunikation.

"Ich persönlich glaube, dass das eine weit wichtigere Frage ist als die Frage, ob die Crowdworker nun Beschäftigte oder Selbstständige sind. Diese Frage hängt letztlich an wenigen Punkten in den AGBs, und wenn die Plattformen die ändern, werden die Beschäftigten sicherlich als Selbstständige eingeordnet werden." (Experte IG Metall)

Aus Gewerkschaftssicht können die Plattformen somit relativ einfach dafür sorgen, dass die in der Crowd Tätigen formalrechtlich als Selbstständige gelten. Unabhängig vom rechtlichen Status der Crowdworker*innen blieben Plattformen aber für die Gestaltung der Arbeitsbedingungen verantwortlich, weshalb den Plattformregeln, z.B. bezogen auf die Offenlegung von Auswahl- und Bewertungskriterien, mehr Beachtung gewidmet werden sollte.

Der dritte Schwerpunkt ist die Mitgliedergewinnung. Zwar hat die IG Metall viele Crowdworker*innen angesprochen und auch einige hundert Gewerkschaftseintritte erreicht. Das ist zwar absolut keine große Zahl; angesichts fehlender betrieblicher Strukturen und betrieblicher Interessenvertretungen - die Mitgliedergewinnung ist das traditionelle Kerngeschäft der Betriebsräte - sind die Erfolge aber auch nicht gering zu bewerten. Dennoch sollen die Ansprache und die Mitgliederwerbung systematisiert werden. Auch dies soll auf der neuen Plattform eingerichtet werden. Zugleich soll die Werbung auf den Kanälen der sozialen Medien intensiviert werden. 
„Dieses Jahr wollen wir unsere systematische Ansprache auf der Plattform verbessern. So dass die Leute sagen: Cool, das ist eine gute Sache, der schließe ich mich an, das ist für mich selber wichtig oder ich kann dazu beitragen, dass es dem Kollektiv besser geht. Dafür wollen wir auch in den Social Media aktiver werden und die entsprechend bedienen." (Experte IG Metall)

\section{Beispiel 3: Selbstständigenberatung von ver.di}

Neben der IG Metall beschäftigen sich in Deutschland auch weitere Gewerkschaften mit den Auswirkungen von Plattformarbeit: In der Dienstleistungsgewerkschaft ver.di stand am Ausgangspunkt der Beschäftigung mit dem Thema die Debatte um das interne Crowdsourcing und die Generation Open bei IBM, die etwa im Jahr 2012 geführt wurde (vgl. Boes, Kämpf, Langes, Lühr, Steglich 2014). IBM hatte erstmals eine Plattform eingerichtet, auf der interne Mitarbeiter untereinander und mit externen Konkurrenten um Projekte konkurrieren sollten (Boewe und Schulten 2016). Ver.di hat dazu ein „Berliner Crowdsourcing-Cloudworking-Papier" verfasst (ver.di 2012), in dem die Wahrung der Interessen von Nutzern und Beschäftigten im digitalen Wandel als Grundlage der Innovationspolitik betont wird. Dann geschah eine Weile wenig, bis sich die Gewerkschaft entschloss, zusammen mit Arbeitswissenschaftlern des ISF München und der Universität Kassel an einem Projekt über Crowdwork mitzuarbeiten, das vom Bundesministerium für Bildung und Forschung (BMBF) finanziert wurde. Die Zielsetzung dabei war aus Sicht der Gewerkschaft, Handlungsempfehlungen für Betriebsräte im Umgang mit Crowdwork zu erarbeiten. Das erwies sich aber als schwierig, weil es keine Informationen gab, welchen Umfang das Phänomen hat, wo im breiten Organisationsbereich der Gewerkschaft es auftaucht und für wen es überhaupt ein Problem ist. Deshalb wurde dann zunächst eine Befragung unter Betriebsräten und Gewerkschaftsmitgliedern durchgeführt.

„Bei der Befragung bei den Gewerkschaftssekretären hat man festgestellt, dass das Thema im Fachbereich 8, also das ist die alte IG Medien und der Bereich Journalismus, angekommen ist. Und im Fachbereich 9, der Telekom und den IT-Dienstleistungen, eben auch über dieses Beispiel IBM. Ansonsten, in der Organisation, aber nicht so richtig wahrgenommen wird." (Expertin 1 ver.di)

Bei der Befragung zeigte sich auch, dass viele Betriebsräte mit dem Begriff der Plattformen noch wenig anfangen konnten und diese häufiger mit Social Media gleichgesetzt haben. Deshalb sieht die Gewerkschaft eine zen- 
trale Aufgabe darin, die Betriebsräte für das Thema als möglicherweise wichtiges Zukunftsfeld zu sensibilisieren; allerdings ohne es zu dramatisieren, denn die Befragung hat auch ergeben, dass die Verbreitung von hauptberuflichem Crowdworking noch gering ist (Pongratz und Bormann 2017).

„Wir machen eine große Tagung für Betriebs- und Personalräte in Berlin, gemeinsam mit der IG Metall, ist wirklich eher eine Sensibilisierung für das Thema. Ohne jetzt zu sagen: Skandal, in wenigen Jahren werden Millionen von Menschen auf diesen Plattformen arbeiten. Sondern schon auch $z u$ sagen: Naja, bis lang sind es relativ wenig, die hauptberuflich da arbeiten." (Expertin 1 ver.di)

Ein weiteres Ergebnis der Befragung war, dass die Auswahl von Aufträgen für die Crowdworker*innen eine wichtige Frage ist und dass überhaupt großer Beratungsbedarf besteht. Deshalb hat sich die Gewerkschaft entschlossen, ein Beratungsangebot durch das Referat für Selbstständige zu organisieren. Das Referat hat traditionell die Betreuung von Journalist"innen übernommen, die häufig als Selbstständige für Verlage, Zeitungen und Zeitschriften arbeiten. Dort gab es deshalb sowohl eine fachliche Expertise für Fragen der selbstständigen Beschäftigung als auch eine hohe Sensibilität für das Thema. Das Referat hat schon länger nicht mehr nur Journalisten betreut, sondern auch andere Gruppen von Selbstständigen.

„Es sind jetzt nicht mehr nur Journalisten, die wir organisieren. Da sind schon noch viele andere Bereiche auch nach und nach dazu gekommen, auch aus anderen Branchen wie dem Bankgewerbe, der Telekommunikation oder auch der Gesundheitsdienstleistungen, wo Pflegekräfte über Plattformen vermittelt werden." (Expertin 1 ver.di)

Das Referat macht inzwischen keine Unterschiede mehr bei der Betreuung zwischen Beschäftigtengruppen. Die zentrale Bedingung lautet, dass es sich um Solo-Selbstständige handelt, die keinen Arbeitnehmerstatus haben und selbst keine anderen Erwerbspersonen beschäftigen. Darauf zielt auch die Ansprache, denn eine Erfahrung aus dem Forschungsprojekt war auch, dass die Crowdworker*innen nicht als Arbeitnehmer*innen angesprochen werden wollen, sondern zu guten Teilen ein Selbstverständnis als Selbstständige aufweisen oder zumindest professionelle Orientierungen haben. Die Anschlussfähigkeit des Referats an die neue Fragestellung war auch deshalb hoch, weil es bereits ein ausgefeiltes Angebot im Internet gab, unter anderem eine Honorardatenbank, und auch ein Selbstständigen-Portal dort verwaltet wurde. Inhaltlich bezieht sich die Beratung auf Honorar-, Branchen-, Sozialversicherungs- oder Vertragsfragen, es wird aber auch 
Rechtsberatung geleistet. Für Gewerkschaftsmitglieder ist die Beratung kostenlos.

„Wir waren da eigentlich schon ganz gut gewappnet und haben sozusagen die Beratung dann eigentlich nur nochmal spezifiziert, eben für Plattformarbeiter." (Expertin 2 ver.di)

Das Referat Selbstständige ist in der Gewerkschaftsorganisation eine eigenständige Organisationseinheit mit einem eigenen Budget. Es betreut derzeit etwa 30.000 Selbstständige, die Mitglieder der Gewerkschaft sind. Ver.di ist allerdings eine komplexe Organisation, die zugleich nach verschiedenen Branchen und Sparten, den Fachbereichen, organisiert ist. In dieser Matrixstruktur ist es auch wichtig, dass fachliche Kompetenzen in den Bereichen wie Finanzdienstleistungen, Telekommunikation oder dem Gesundheitswesen selbst verankert sind. Das Referat Selbstständige kooperiert mit den Fachbereichen, aber echte personelle Zuständigkeiten für das Thema gibt es dort noch nicht. In jedem Fall gibt es mit Blick auf die Organisierung von Selbstständigen noch einiges zu tun.

„Wir sind 30.000 Mitglieder, Selbstständige, bei ver.di. Das konnte konstant gehalten werden, was, wenn man die Entwicklung der der Gesamtorganisation anguckt, gut ist. Aber es gibt rund 2,1 Millionen Solo-Selbstständige circa in Deutschland. Also wir haben noch Potenzial." (Expertin 1 ver.di)

Bei der Organisierung stellt sich für die Gewerkschaft freilich auch die Frage von Kosten und Ertrag. Dies gilt insbesondere für die Crowdwork, dessen quantitative Bedeutung gerade mit Blick auf Beschäftigte mit Hauptverdienst auf Plattformen offensichtlich eher über- als unterschätzt wurde. Online-Beschäftigte (Cloudworker*innen) sind aus Sicht der Expertin erstens weniger zahlreich als Offline-Plattformbeschäftigte (Gigworker"innen) beispielsweise im Gesundheitsbereich, und sie sind schlechter erreichbar und damit auch schlechter organisierbar.

„Weil meine Erkenntnisse aus dem Projekt wären eigentlich, dass die quantitative Bedeutung überschätzt wurde und dass man schon auch wirklich die Frage sich stellen muss, als ver.di oder auch als IG Metall: Wie viel Ressourcen steckt man in die Organisierung von Crowdworkern rein? Die Hürde ist nicht, dass sie Selbstständige sind, sondern die Hürde ist der Online-Charakter. Von den Strategien ist es auch für Gewerkschaften wichtig zu unterscheiden." (Expertin 1 ver.di)

Unabhängig davon versucht auch ver.di, ähnlich der IG Metall, Gespräche mit den Plattformen aufzunehmen, um auf diese Weise Einfluss auf die Arbeitsbedingungen ausüben zu können. Das Ziel, mit Plattformen Haus- 
tarifverträge abzuschließen, steht zwar noch in weiter Ferne. Echte Verhandlungen gibt es noch nicht. Dennoch aber werden Gespräche zumindest mit einigen Plattformen geführt, die ihren Sitz in Deutschland haben; sie zeigen sich zumindest aufgeschlossen, mit der Gewerkschaft zu reden. Dabei geht es um Informationsaustausch, die Frage möglicher gemeinsamer Ziele und auch die Frage, wie und ob verbindlichere Regelungen zu den Arbeitsbedingungen möglich sind.

„So die Zielsetzung ist erstmal teilweise überhaupt sich kennen zu lernen. Dass die dann vielleicht erstmal wissen, was eigentlich Gewerkschaften machen und welche Ziele wir verfolgen [...]. Und die erklären uns erstmal, wie ihr Geschäftsmodell ist, wie es vielleicht auch aussieht mit der Transparenz [...]. Und dann natürlich auch so ein bisschen zu auszuloten, inwiefern man vielleicht auch zu gemeinsamen politischen Forderungen kommen kann. Also wenn die Plattformbetreiber auch gar kein Problem damit haben, sich zum Beispiel für so eine Erwerbstätigenversicherung einzusetzen, die Plattformarbeiter in die Sozialversicherung einbindet." (Expertin 2 ver.di)

\section{Beispiel 4: Erste Ansätze einer organisierten Interessenartikulation bei Blogger*innen und Vlogger"innen}

Das Gigwork und das aus dem on-demand Bearbeiten von (insbesondere Kleinst-)Arbeitsaufträgen bestehende Crowdwork haben somit bereits einige wissenschaftliche Aufmerksamkeit bekommen (vgl. Leimeister et al. 2016; Huws, Spencer, Syrdal und Holts 2017; Lehdonvirta et al. 2018). Das obige Beispiel der Verknüpfung von Plattformarbeit und Selbstständigenberatung deutet aber bereits darauf hin, dass sich in der Plattformökonomie neue Formen der Selbstständigkeit herausbilden, bei denen abseits klassischer Berufsfelder neue Arbeitsformen im und durch das Internet entstehen. Diese sind bislang aus soziologischer Perspektive allerdings erst in Ansätzen untersucht worden (Papsdorf 2018). Abschließend soll nun noch ein Blick darauf gelenkt werden, ob und inwieweit es in diesen Arbeitsformen überhaupt einen Austausch über gemeinsame Interessenlagen gibt und inwiefern sich Ansätze einer Organisation derartiger Anliegen herausbilden.

Eine durch die Digitalisierung möglich gemachte Form von Solo-Selbstständigkeit, ist diejenige von Blogger*innen und Vlogger*innen (VideoBlogger*innen). Die Weiterentwicklung von digitalen und mobilen Kommunikationsmedien erweist sich für diese Selbstständigen nicht nur als 
eine technische Infrastruktur über die Informationen bezogen und Daten ausgetauscht werden können, sondern stellt vielmehr einen neuen „sozialen Handlungsraum" (Boes 1996) dar, in dem Menschen auf unterschiedlicher Weise miteinander interagieren können und der neue Handlungsmöglichkeiten sowie neue Formen des Wirtschaftens erlaubt (Boes, Kämpf, Langes und Ziegler 2017, S. 64-66). Die sich in den letzten Jahren vollziehende Weiterentwicklung des Internets, durch die es größeren Nutzerkreisen ermöglicht wird, eine aktive Rolle bei der Erstellung von Inhalten zu spielen (sogenannter user generated content), stellt eine zentrale Grundlage für die Herausbildung neuer, internetbasierter Arbeitsformen dar (Papsdorf 2018). Zudem bestehen hierbei vergleichsweise geringe Eintrittsbarrieren, da kaum Betriebskapital oder spezifische Berufszertifikate notwendig sind, um Erwerbszecke zu verfolgen.

Blogs und Vlogs sind in den vergangenen Jahren sowohl international als auch in Deutschland zu einem zunehmend bedeutenden Phänomen geworden (Schmidt 2018, S.9-11.; Taddicken und Schmidt 2017, S. 13-18). Die genaue Zahl von Blogs und Vlogs lässt sich aufgrund eines fehlenden Gesamtverzeichnisses und der hohen Dynamik in diesem Feld allerdings nicht exakt bestimmen (zur Messproblematik vgl. Schmidt 2006, S. 15-21; Schenk, Niermann und Briehl 2014, S.4). Verschiedene Schätzungen, die auf Angaben von Bloghostern basieren, gehen von mehr als 200.000 aktiven Blogs in Deutschland aus. ${ }^{10}$ Es ist jedoch weitgehend unbekannt, wie viele dieser Blogs erwerbsmäßig von Solo-Selbstständigen betrieben werden. Hinzu kommen im Feld der Blogger"innen noch all jene Personen, die keinen eigenen Blog betreiben, sondern stattdessen einen oder mehrere sogenannte ,Kanäle ${ }^{6}$ auf diversen Social-Media-Plattformen wie YouTube, Instagram, Pinterest oder auch Facebook betreiben. Während über die Zahl der haupt- und nebenberuflichen Blogger*innen wenig Wissen vorliegt, deuten zumindest die Nutzerstatistiken der genannten Plattformen darauf hin, dass sich hier ein durchaus lukratives Erwerbsfeld entwickelt hat: Die Abrufstatistiken von YouTube, dem weltweit beliebtesten

10 Verschiedene Blogger haben in Beiträgen versucht, die Zahl deutscher Blogs oder die Zahl der Blogger*innen aus Deutschland zu bestimmen. Auch weil sich der Markt der Bloghoster sehr dynamisch zeigt, ist die Qualität der vorliegenden Daten jedoch eingeschränkt. Allerdings scheint die Zahl der geführten Blogs tendenziell weiter anzusteigen (vgl. hierzu die Blogbeiträge von Jan Schmidt (http://ww w.schmidtmitdete.de/archives/707), Sven Lennartz (https:/conterest.de/wie-vieleblogs-gibt-es-zahlen-statistiken/) oder Christian Buggisch (https://buggisch.wordp ress.com/2016/02/23/wie-viele-blogs-gibt-es-in-deutschland/); jeweils zuletzt abgerufen am 4.10.2018). 
Video-Portal, dokumentieren beispielsweise, dass Vlogs deutscher YouTuber*innen millionenfach angeschaut werden. Auch die Ergebnisse der „ARD/ZDF-Online Studie 2017“ zeigen, dass insbesondere bei Jugendlichen und jungen Erwachsenen Vlogs beliebt sind. So schaut laut der ARD/ZDF-Online-Studie mittlerweile über die Hälfte der 14-19-jährigen täglich Videos auf Videoportalen wie YouTube (Kupferschmitt 2017). Blogs und Vlogs bieten aufgrund dieser Nutzer*innenzahlen verschiedene Möglichkeiten, um mit dem Erstellen von Bloginhalten Einnahmen zu generieren. Typisch ist dabei, dass häufig von den Konsumenten keine Gebühren erhoben werden, sondern dass die mit den Blogs erzielte Aufmerksamkeit mittels präsentierter Werbung in ökonomisches Kapital transformiert wird (Reichert 2008, S. 62-65).

Aktuelle Forschungsergebnisse aus dem Projekt „Bloggen „Entgrenzte Arbeit im Netz: Bloggen und Vloggen als neue digitale Arbeitsformen“ (Hoose und Rosenbohm 2020) ${ }^{11}$ zeigen, dass sich die Einnahmequellen von Blogger"innen häufig aus verschiedenen Quellen speisen und das Bloggen als Erwerbsarbeit dadurch verschiedene Tätigkeiten und auch Beschäftigungsformen miteinander verknüpft. Es zeigt sich, dass sich die durch digitale Technologien ermöglichten Selbstständigkeiten als fragmentierte Erwerbstätigkeiten präsentieren, in denen Einnahmen aus selbstständiger Arbeit auch mit solchen aus abhängiger Beschäftigung kombiniert werden können (Hoose 2019). In der soziologischen Forschung zu Selbstständigkeit werden derartige Entwicklungen auch als Hybridisierung von Erwerbsformen (vgl. Bührmann, Fachinger und Welskop-Deffaa 2018) gefasst.

Insbesondere, wenn digitale Solo-Selbstständige sehr mobil arbeiten, werden sie häufig als, digitale Nomad"innen' (Nash, Jarrahi, Sutherland und Phillips 2018) bezeichnet. Dabei handelt es sich um reiseinteressierte, abenteuerlustige und technologieaffine junge Wissensarbeiter"innen, die ein Einkommen aus Tätigkeiten zum Beispiel als (Reise)Blogger*in, aus Aktivitäten auf Social-Media-Plattformen wie Instagram und YouTube sowie aus weiteren Freelancer*innen-Tätigkeiten erzielen und dabei keinen festen Arbeits- und Lebensmittelpunkt haben (Müller 2016). Digitale Technologien spielen für diese Art des mobilen Arbeitens eine besondere Rolle, denn erst digitales Arbeiten und die Übermittlung von Arbeitsergebnissen via Internet ermöglicht derartige Arbeits- und Lebensmodelle. Dabei werden diverse Online-Plattformen und (Kommunikations-)Tools

11 Für weitere Infos zum Projekt siehe https://www.uni-due.de/iaq/projektinfo.php? $\mathrm{p}=\mathrm{B} \log \mathrm{A}$. 
einerseits zur Koordination der verschiedenen Arbeitstätigkeiten genutzt. Darüber hinaus nutzen digitale Nomad"innen die gleichen Werkzeuge jedoch auch für die Organisation dieses Lebensstils. In diesem Sinne haben sich verschiedene Online-Plattformen und Internet-Foren (z.B. nomadforum.io; vgl. Nash et al. 2018, S. 208) gebildet. Diese Plattformen dienen nicht allein dem Austausch und der Information über lohnende Reiseziele; darüber hinaus etabliert sich hier auch der Informationsaustausch über Beschäftigungsmöglichkeiten und es lassen sich beispielsweise Kooperationen zwischen verschiedenen digitalen Nomaden anbahnen. Zusätzlich dienen diese Plattformen auch dem Austausch über Arbeitsbedingungen und verschiedene Belange der Organisation von Selbstständigkeit (z.B. Fragen der benötigten (Sozial-)Versicherungen; rechtliche Aspekte der mobilen Arbeit in verschiedenen Ländern etc.).

Ähnliche Informations- und Austauschmöglichkeiten entstehen auch für weitere Spielarten digitaler Solo-Selbstständiger. Gerade weil hier die Übergänge zwischen Freizeitaktivitäten und bezahlter Arbeit häufig fließend sind, stellen sich hier für professionelle Blogger*innen besondere Herausforderungen ein: Teils bezahlte Arbeitsleistungen werden hier mit weiteren Tätigkeiten kombiniert, die jedoch als eher flankierende Handlungen mit primärem Freizeitbezug zu charakterisieren sind (Hoose 2019). Weil aber Blogger*innen, die von dieser Tätigkeit leben möchten, ein Interesse daran haben, dass diese Tätigkeit auch von Konsument*innen und insbesondere von Sponsor*innen als professionelle Arbeit anerkannt wird, gibt es seit 2013 einen sogenannten „Blogger-Kodex“12. Mit diesem Kodex verpflichten sich Blogger*innen unter anderem zum transparenten Umgang mit Kooperationen oder zur Einhaltung inhaltlicher Qualitätsstandards. Nachdem der Blogger-Kodex als Initiative einiger Reiseblogger*innen gestartet wurde, ${ }^{13}$ sind hier mittlerweile auch weitere Blogger*innen zum Beispiel aus den Bereichen Ernährung, Fotografie oder Familie/Eltern versammelt. Unterzeichner"innen des Kodex werden auf der Webseite des Blogger-Kodex in einem Verzeichnis aufgelistet und signalisieren zudem ihre Mitgliedschaft ${ }^{14}$ auf ihren Blogs und Social-Media-Kanälen.

12 vgl. http://reiseblogger-kodex.com/reiseblogger-kodex/; zuletzt abgerufen am 30.10.2019.

13 Weshalb die Initiative ursprünglich auch als „Reiseblogger-Kodex“ gestartet wurde.

14 Wer in die Liste der Kodexunterzeichner*innen aufgenommen werden möchte, muss sich hierfür bewerben. Bedingungen für die Aufnahme sind die Zahlung einer Aufnahmegebühr von 50 Euro, das Bestehen des Blogs seit mindestens drei 
In diesen Bereich neuer digitaler Einkommensmöglichkeiten sind darüber hinaus erste Anzeichen für eine Organisation von Interessen zu finden. So hat sich im Jahr 2016 der Bloggerclub e.V. gegründet, der sich selbst zum Anspruch setzt „eine Interessenvertretung für unsere Mitglieder [...] und idealerweise mittelfristig für alle deutschen Blogger" ${ }^{\text {"15 }} \mathrm{zu}$ sein. Neben der Bereitstellung von Online-Informationen sind bei diesen Versuchen der Organisation kollektiver Interessen von digitalen Solo-Selbstständigen Bemühungen erkennbar, den virtuellen Austausch im Internet auch um reale Vernetzungstreffen zu erweitern: Im Falle des Bloggerclubs geschieht dies durch die Ausrichtung von „Bloggerstammtischen“ sowie Workshops und Vorträgen. Darüber hinaus etablieren sich in der Blogger*innen- und auch der Digitalen Nomaden-Szene verschiedene Konferenzen als Austausch- und Informationsveranstaltungen rund um die Tätigkeiten dieser Formen von Solo-Selbstständigkeit. Es bleibt abzuwarten, inwieweit die Gestaltung der Arbeitsbedingungen in derartigen Austauschformaten zukünftig eine Rolle spielen wird.

Ein Beispiel für eine solche Weiterentwicklung des Austausches über ,Arbeitsbedingungen' stellt die die Anfang 2018 gestartete Initiative „Youtubers Union“ 16 dar. Ausgangspunkt für die Initiative war die Unzufriedenheit von auf dieser Plattform aktiven professionellen YouTuber*innen mit den Plattformregeln. Eine Einnahmequelle von YouTuber*innen besteht darin, dass vor von ihnen erstellten Videos Werbung gezeigt wird. Professionelle YouTuber*innen können ihre Beiträge monetarisieren: Das bedeutet, dass sie von der Plattform YouTube an den Werbeeinnahmen beteiligt werden. Je häufiger ein Video angesehen wird, desto höher fallen dementsprechend die Einnahmen für die YouTuber"innen aus. Im Jahr 2017 änderte die Plattform aber die bis dahin bestehenden Regeln für die Monetarisierung. So wurden insbesondere die Themen von Videos, mit denen eine Monetarisierung fortan möglich war, neu gefasst. Mit Videos $\mathrm{zu}$ von nun an als werbeunfreundlich geltenden Inhalten (darunter fallen z.B. Inhalte in Bezug auf Schusswaffen, in Bezug auf Drogen oder auch Videos in denen kontroverser Themen und sensible Ereignisse behandelt werden) konnte daher kein Geld mehr verdient werden ${ }^{17}$.

Monaten sowie eine Prüfung des Blogs durch die Kodex-Initiator*innen (vgl. http://reiseblogger-kodex.com/mitmachen/; zuletzt abgerufen am 30.10.2019).

15 https://www.bloggerclub.de/der-bloggerclub/; zuletzt abgerufen am 15.03.2019.

16 vgl. https://youtubersunion.org/; zuletzt abgerufen am 01.10.2019.

17 Die „Richtlinien für werbefreundliche Inhalte“ (https://support.google.com/yout ube/answer/6162278; zuletzt abgerufen am 14.10.2020) lassen jedoch Platz für 
Als Reaktion auf diese Veränderungen organisierten sich einige YouTuber*innen über verschiedene Gruppen in sozialen Netzwerken. Initial waren hierfür Videos des YouTubers Jörg Sprave, in denen er zur Gründung einer YouTuber-Gewerkschaft aufrief..$^{18}$ Die Initiative fordert insbesondere mehr Transparenz von YouTube hinsichtlich der selbst gesetzten Werbe-Regeln und auch hinsichtlich der genutzten Plattformalgorithmen. Über die Algorithmen werden zum Beispiel Videos prominenter präsentiert und erreichen damit mehr oder weniger Zuschauer"innen, was sich dann wiederrum auf die Einnahmemöglichkeiten der YouTuber*innen auswirkt. Zwar konnte die Initiative damit einige öffentliche Aufmerksamkeit erregen und zählte selbst mehr als 15.000 Unterstützer"innen, konnte jedoch im Gespräch mit der Plattform keine konkreten Erfolge erzielen (Niebler 2019). Seit Juli 2019 wird die Initiative von der IG Metall unterstützt, mit der gemeinsam die Kampagne „FairTube“ gestartet wurde (IG Metall 2019). Dabei wurde unter anderem damit argumentiert, dass die Video-Plattform den aktiven professionellen Blogger*innen so konkrete Regeln für ihre Arbeit setzt, dass es sich bei ihnen letztlich um Schein-Selbstständige handele. Nach einigen gescheiterten Gesprächsversuchen wurde die Kommunikation zwischen Plattform und Gewerkschaft wiederaufgenommen. Als Hauptanliegen werden nun die Transparenz und Nachvollziehbarkeit von Plattformentscheidungen genannt ${ }^{19}$. Zudem wird, nach Vorbild der Ombudsstelle im Bereich Crowdwork (vgl. Abschnitt 4) auch für die YouTuber*innen die Einrichtung einer unabhängigen Schlichtungsstelle gefordert.

Deutlich wird in der Betrachtung der obigen Beispiele: Nicht nur die Formen von Arbeit und ihrer Koordinierung wandeln sich mit den neuen Möglichkeiten digitaler Technologien - auch die Möglichkeiten der Interessenvertretung verändern sich analog dazu. Das birgt Herausforderungen für tradierte Interessenvertretungsstrukturen, wenn hier neue Konkurrenz auf den Plan tritt. Das eröffnet aber andererseits auch Wege hin in Bereiche von Arbeit und Beschäftigung, die bislang für die etablierten Akteure der Interessenvertretung verschlossen waren.

unterschiedliche Interpretationen, wodurch Demonetarisierungsentscheidungen der Plattform z.T. als unberechtigt empfunden werden.

18 Siehe https://www.youtube.com/watch?v=q7RfYt_p2mk\&feature=emb_title (zuletzt abgerufen am 24.04.2020).

19 vgl. https://www.fairtube.info/de/, zuletzt abgerufen am 24.04.2020. 


\section{Fazit}

Die oben exemplarisch aufgeführten Beispiele zeigen - bei allen Einschränkungen und widersprüchlichen Erfahrungen - was die Erfolgsaussichten derartiger Initiativen betrifft: Der Bereich der Plattformarbeit mag vorrausetzungsvoll für die Artikulation kollektiver Interessen sein, aber die konkurrenzfördernde Plattformlogik verhindert das Entstehen gemeinsamer Arbeitnehmeranliegen nicht. Anknüpfungspunkte dafür bietet die Plattformarbeit mit ihren fragmentierten Beschäftigungsbedingungen, niedrigen Entgelten und dichten Kontrollstrategien genug. Wie gezeigt werden konnte, bestehen deshalb bei den sich neu entwickelnden Arbeitsformen Ansprüche an kollektiven Interessenaustausch. Ein Bedarf dafür ist selbst bei sehr individualisierten und in jeweils sehr unterschiedlichen Arbeitszusammenhängen eingebundenen Solo-Selbstständigen, wie professionellen Blogger*innen, vorhanden. Mit digitalen Kommunikationskanälen können niedrigschwellige Informationsangebote geschaffen und von den so Arbeitenden selbst initiiert werden. Die Nutzung derartiger Kommunikationsmöglichkeiten ist dabei von einer ,Organisation von Arbeitnehmerinteressen' aber (bislang noch) ein ganzes Stück weit entfernt.

Eine zentrale Voraussetzung dafür ist das Vorhandensein von Initiativen, die Informationen sammeln, Dialog in Gang setzen, Beteiligung organisieren und auf diese Weise versuchen, gemeinsame Interessen zu entwickeln und zu konstruieren. Obwohl die Hauptverantwortung für die Gestaltung und Regulierung von Plattformarbeit, insbesondere in Form von Crowd- und Gigwork, häufig vor allem bei politischen Rahmensetzungen gesehen wird (Schönefeld und Hensel 2019, S. 23-24, Baethge, Borberach, Hoffmann und Wintermann 2019, S. 66), verlassen sich die hier vorgestellten Initiativen (und dies ist insbesondere bei der Initiative Fair Crowd Work der IG Metall zu konstatieren) nicht auf zukünftige Rechtsänderungen. Zwar sind solche Änderungen derzeit (noch) nicht konkret angedacht, der Bedarf dafür wird aber gerade durch die Initiativen deutlich. Dazu gehört die Frage der rechtlichen Absicherung befristet beschäftigter Betriebsräte ebenso wie der Schutz von Initiator*innen bei Betriebsratswahlen vor der Wahl des Wahlgremiums oder auch die Frage der Betriebsförmigkeit von Plattformarbeit.

Die Initiativen erfolgen sowohl Bottom-Up als auch Top-Down. Die Bottom-Up-Initiative „Liefern am Limit“ der Rider profitierte von der Ortsabhängigkeit des Gigworkings. Die Fahrradkuriere konnten sich während ihrer Arbeit (sowie in Pausen oder auf dem Weg vom/zum Schichtbeginn) im Stadtbild treffen und anhand ihrer Kleidung auch erkennen. So kommt es, dass die Rider sich auch während ihrer Arbeitszeit direkt mit 
Kolleg*innen austauschen können (Heiland 2019, S. 301). Daraus lässt sich schließen, dass für die solidarische Artikulation von Interessen auch bei digital koordinierter Arbeit direkte Kommunikation und der unmittelbare Austausch - also das Gespräch von Angesicht zu Angesicht - wichtig bleiben. Wo sie bestehen, ist eine emergente Artikulation durch lokale Initiativen möglich, auch wenn sie durch prekäre Beschäftigungsformen erschwert werden. Deshalb ist, wie das Beispiel zeigt, die Hilfe durch etablierte Gewerkschaftsorganisationen wichtig, um den Initiativen Ressourcen zukommen zu lassen und Kontinuität zu ermöglichen.

Dies gilt umso mehr im Bereich des Cloudworking. Hier steht das Fehlen dieser Kopräsenz von Crowdworker*innen etwaigen Bottom-Up Initiativen entgegen. Dennoch gibt es derartige Ansätze auch hier, wie der Verweis auf das Projekt Turkopticon zeigen konnte. Ungleich mehr Gewicht erhalten solche Initiativen jedoch, wenn sie die organisatorische Unterstützung durch etablierte Interessenvertretungsorganisationen Top-Down erhalten. In räumlich atomisierten Beschäftigungsverhältnissen bedarf es einer organisatorischen Artikulation, die von etablierten Interessenvertretungen ausgeht. Voraussetzung für ein solches Engagement ist freilich, dass sich in der Abwägung von Aufwand und Ertrag solcher Initiativen die Ansicht durchsetzt, derartige Interventionen könnten wichtige Zukunftsinvestitionen für Gewerkschaften sein. Die wechselhafte Historie der Selbstständigenberatung der Gewerkschaft ver.di deutet an, dass eine Verständigung darauf jedoch nicht immer leicht gegeben ist; gerade weil beispielsweise noch zu sehr auf (derzeit) wenige hauptberufliche Crowdworker"innen fokussiert wird, die zudem in Beschäftigungsverhältnissen tätig sind, die denen der klassischen Kernzielgruppen von Gewerkschaften so gar nicht entsprechen. Die Methoden, mit denen Bottom-Up-Initiativen wie die vorgestellten zwangsläufig experimentieren, können hierbei gewerkschaftliches Handeln insgesamt beeinflussen (vgl. Vandaele 2018).

Ein drittes Feld der Initiativen bilden schließlich die Blogger"innen und Vlogger"innen, die eher Unternehmer*innen in eigener Sache als Beschäftigte sind. Das Engagement einzelner Protagonist"innen und die jeweils artikulierten Themenschwerpunkte der Interessen sind hier sehr viel situativer und thematisch vielfältiger als in traditionellen Vertretungsformen üblich. Die vorgestellten Beispiele digitalen Interessenaustausches scheinen dabei Ausdruck einer „subjektivierten Solidarität" (Hoose und Schütte 2013) unter Arbeitenden zu sein, die zwar in „[u]nverbindlicheren und kurzfristigeren Konstellationen" (Lee und Staples 2018, S. 502) mündet, sich jedoch als dauerhaftes Phänomen in den neuen Erwerbsformen etablieren könnte. 
Folgt man der Ansicht, die Entwicklung der Plattformökonomie werde künftig immer mehr Ausstrahlungseffekte in traditionelle Bereich von Erwerbsarbeit entfalten, dann bekommen die betrachteten Initiativen eine Bedeutung über ihre konkreten Anliegen (wie z.B. der Verbesserung der Arbeitsbedingungen bei Essenslieferdiensten) hinaus: An diesen neuen Formen der emergenten und organisierten Interessenartikulation kann wie in einem Brennglas studiert werden, welche Möglichkeiten der Konstruktion und Formung kollektiver Interessen es auch bei digitaler Arbeitsorganisation gibt und wie digitale Kommunikationstechnologien nicht nur im Sinne von Vermarktlichung, Effektivitätssteigerungen oder erweiterter Prozesskontrolle, sondern auch für die Entwicklung kollektiver Interessen und die Aushandlung von guten Arbeitsbedingungen für digital Arbeitende genutzt werden können.

\section{Literatur}

Altenried, M. (2017). Die Plattform als Fabrik: Crowdwork, Digitaler Kapitalismus und die Vervielfältigung der Arbeit. PROKLA, 47(187), 175-191.

Altenried, M., Niebler, V., \& Wallis, M. (2020). On-demand. Prekär. Systemrelevant. der Freitag (17/2020). Verfügbar unter: https://www.freitag.de/autoren/der-f reitag/on-demand-prekaer-systemrelevant [24.04.2020].

Baethge, C. B., Borberach, M., Hoffmann, A., \& Wintermann, O. (2019). Plattformarbeit in Deutschland: Freie und flexible Arbeit obne soziale Sicherung. Gütersloh. Verfügbar unter: https://www.bertelsmann-stiftung.de/fileadmin/files/BSt/Publi kationen/GrauePublikationen/Plattform_.pdf [09.05.2019].

Bauer, R. M., \& Gegenhuber, T. (2015). Crowdsourcing: Global search and the twisted roles of consumers and producers. Organization, 22(5), 661-681.

Benner, C. (2014a). Amazonisierung oder Humanisierung der Arbeit durch Crowdsourcing? In C. Benner (Hrsg.), Crowdwork - zurück in die Zukunft? Perspektiven digitaler Arbeit (S. 289-300). Frankfurt: Bund Verlag.

Benner, C. (Hrsg.) (2014b). Crowdwork - zurück in die Zukunft? Perspektiven digitaler Arbeit. Frankfurt: Bund-Verlag.

Bertschek, I., Ohnemus, J., \& Viete, S. (2015). Befragung zum sozioökonomischen Hintergrund und zu den Motiven von Crowdworkern: Endbericht zur Kurzexpertise für das Bundesministerium für Arbeit und Soziales. Mannheim. Zentrum für Europäische Wirtschaftsforschung GmbH (ZEW).

Boes, A. (1996). Formierung und Emanzipation: Zur Dialektik der Arbeit in der „Informationsgesellschaft“". In R. Schmiede (Hrsg.), Virtuelle Arbeitswelten: Arbeit, Produktion und Subjekt in der "Informationsgesellschaft" (S. 159-178). Berlin: edition sigma. 
Boes, A., Kämpf, T., Langes, B., Lühr, T., \& Steglich, S. (2014). Cloudworking und die Zukunft der Arbeit: Kritische Analysen am Beispiel der Strategie "Generation Open" von IBM. Kassel: BTQ Kassel.

Boes, A., Kämpf, T., Langes, B., \& Ziegler, A. (2017). Unternehmen und die Cloud. Arbeit, 26(1), 61-86.

Boewe, J., \& Schulten, J. (2016). Die Macken der Prototypen. MITBESTIMMUNG (02/2016), 27-29.

Börner, F., Kehl, C., \& Nierling, L. (2017). Chancen und Risiken mobiler und digitaler Kommunikation in der Arbeitswelt: Endbericht zum TA-Projekt (Arbeitsbericht Nr. 174). Berlin. Büro für Technikfolgen-Abschätzung beim deutschen Bundes$\operatorname{tag}(\mathrm{TAB})$.

Bührmann, A. D., Fachinger, U., \& Welskop-Deffaa, E. M. (Hrsg.). (2018). Hybride Erwerbsformen. Wiesbaden: Springer.

Däubler, W. (2015). Internet und Arbeitsrecht: Web 2.0, Social Media und Crowdwork (5. Aufl.). Recht aktuell. Frankfurt: Bund-Verlag.

Dickel, S., \& Thiem, C. (2018). Zur Organisation von Arbeit 4.0: Crowdsourcing als Sozialtechnologie. In T. Redlich, M. Moritz \& J. P. Wulfsberg (Hrsg.), Interdisziplinäre Perspektiven zur Zukunft der Wertschöpfung (S. 247-254). Wiesbaden: Springer.

Drahokoupil, J., \& Fabo, B. (2016). The platform economy and the disruption of the employment relationship. ETUI Policy Brief 5/2016. Brüssel: ETUI. Verfügbar unter: https://www.etui.org/content/download/23770/197926/file/Platform+ economy+Drahokoupil+Fabo+Policy+Brief $+\mathrm{PB}+2016.05$.pdf [16.05.2019].

Dolata, U. (2020). Plattform-Regulierung. Koordination von Märkten und Kuratierung von Sozialität im Internet. Berliner Journal für Soziologie, 29(3-4), 179-206.

Ellmer, M. (2016). „Ich geb' dir eine Review, Große/r!“: Amazon Mechanical Turk, Turkopticon und Solidarität in transnationalen, digitalen Arbeitsräumen. Kurswechsel (2), 51-62.

Erdsiek, D., Ohnemus, J., \& Viete, S. (2018). Crowdworking in Deutschland 2018: Ergebnisse einer ZEW-Unternehmensbefragung: Expertise im Auftrag des Bundesministeriums für Arbeit und Soziales. Mannheim. Zentrum für Europäische Wirtschaftsforschung GmbH (ZEW). Verfügbar unter; https://www.bmas.d e/SharedDocs/Downloads/DE/PDF-Publikationen/Forschungsberichte/fb525-cro wdworking-in-deutschland-2018.pdf?_blob=publicationFile\&v=1 [27.02.2019].

Gerber, C., \& Krzywdzinski, M. (2017). Schöne neue Arbeitswelt? Durch Crowdworking werden Aufgaben global verteilt. WZB Mitteilungen (155), 6-9.

Gerber, C., \& Krzywdzinski, M. (2019). Entgrenzung in der digitalen Onlinearbeit am Beispiel von Crowdwork. In H. Hanau \& W. Matiaske (Hrsg.), Entgrenzung von Arbeitsverhältnissen: Arbeitsrechtliche und sozialwissenschaftliche Perspektiven (S. 25-47). Baden-Baden: Nomos.

Greef, S., \& Schroeder, W. (2017). Plattformökonomie und Crowdworking: Eine Analyse der Strategien und Positionen zentraler Akteure (Forschungsbericht Nr. 500). Berlin. Bundesministerium für Arbeit und Soziales (BMAS). 
Harmon, E., \& Silberman, M. S. (2018). Rating Working Conditions on Digital Labor Platforms. Computer Supported Cooperative Work, 37(3), 1275-1324.

Heiland, H. (2019). Plattformarbeit im Fokus. WSI-Mitteilungen, 72(4), 298-304.

Heiland, H., \& Brinkmann, U. (2020). Liefern am Limit. Wie die Plattformökonomie die Arbeitsbeziehungen verändert. Industrielle Beziehungen, 27(2), 120-140.

Hoffmann, R., \& Suchy, O. (2016). Aussichten für die Arbeit der Zukunft (Working Paper Forschungsförderung 013, Mai 2016). Düsseldorf. Verfügbar unter: https://www.boeckler.de/pdf/p_fofoe_WP_013_2016.pdf [07.05.2019].

Hoose, F. (2019). Nur noch das machen, was Spaß macht: Auf der Suche nach der Digitalisierungsdividende für solo-selbständige Kreative. Beitrag zur Ad-HocGruppe »Zwischen Profession und Publikum: Zur Gesellschaftstheorie des Amateurismus«. In N. Burzan (Hrsg.), Komplexe Dynamiken globaler und lokaler Entwicklungen. Verhandlungen des 39. Kongresses der Deutschen Gesellschaft für Soziologie in Göttingen. Verfügbar unter: http://publikationen.soziologie.de/index.p hp/kongressband_2018/article/view/1007.

Hoose, F., \& Rosenbohm, S. (2020). (Video-)Bloggen als Plattformarbeit? Konturen einer Form digitalen Arbeitens (IAQ-Report 2020-11). Duisburg: Insitut Arbeit und Qualifikation.

Hoose, F., \& Schütte, P. (2013). Fragmentierte Solidarität. Das Ende des organischen Zusammenhaltes durch subjektivierte Erwerbsarbeit. Arbeits- und Industriesoziologische Studien, 6(1), 49-63.

Howe, J. (2006). The Rise of Crowdsourcing. Wired Magazin, 14(6). Verfügbar unter: https://www.wired.com/2006/06/crowds/ [04.05.2018].

Huws, U. (2016). Logged labour: A new paradigm of work organisation? Work Organisation, Labour \& Globalisation, 10(1), 7-26.

Huws, U., Spencer, N. H., Syrdal, D. S., \& Holts, K. (2017). Work in the European gig economy: Research results from the UK, Sweden, Germany, Austria, The Netherlands, Switzerland and Italy. Brussels, Hatfield. FEPS; UNI Europa; University of Hertfordshire. Verfügbar unter: https://www.feps-europe.eu/Assets/Pu blications/PostFiles/579_1.pdf [16.05.2019].

IG Metall (2019). Presseinformation: IG Metall und YouTubers Union vereinbaren Zusammenarbeit: Gemeinschaftsprojekt für faire Arbeitsbedingungen im Bereich Video-Crowdworking. Verfügbar unter: https://www.igmetall.de/presse/pr essemitteilungen/ig-metall-youtubers-union-vereinbaren-zusammenarbeit [04.10.2019].

IG Metall, Dänische Gewerkschaft der Vertriebs- und Büroangestellten, International Brotherhood of Teamsters, Local 117, Kammer für Arbeiter und Angestellte, Service Employees International Union \& Unionen. (2016). Frankfurter Erklärung zu plattformbasierter Arbeit: Vorschläge für Plattformbetreiber, Kunden, politische Entscheidungsträger, Beschäftigte und Arbeitnehmerorganisiationen. Verfügbar unter: https:/www.igmetall.de/download/20161214_Frankfurt_Paper _on_Platform_Based_Work_DE_1c33819e1e90d2d09e531a61a572a0a423a93455 . $\operatorname{pdf}[30.10 .2019]$. 
Kaiser, S., Süß, S., \& Josephs, I. (2012). Das Phänomen Freelancing: Eine neue flexible Beschäftigungsform und ihre Herausforderungen. In S. Kaiser, S. Süß \& I. Josephs (Hrsg.), Forschung und Praxis zukunftsfähiger Unternehmensführung: $B d .2$. Freelancer als Forschungsgegenstand und Praxisphänomen: Betriebswirtschaftliche und psychologische Perspektiven (S. 7-18). Frankfurt am Main: Peter Lang.

Kirchner, S. (2019). Arbeiten in der Plattformökonomie: Grundlagen und Grenzen von „Cloudwork“ und „Gigwork“. KZfSS Kölner Zeitschrift für Soziologie und Sozialpsychologie, 71(1), 3-25.

Kirchner, S., \& Beyer, J. (2016). Die Plattformlogik als digitale Marktordnung. Zeitschrift für Soziologie, 45(5), 324-339.

Klebe, T. (2017). Arbeitsrecht 4.0: Faire Bedingungen für Plattformarbeit (WISO direkt 22/2017). Bonn: Friedrich-Ebert-Stiftung.

Kramer, P.-F. (2018). Interessenvertretung in der Wolke? Historische Erfahrungen und aktuelle Herausforderungen der Erwerbsregulierung bei Solo-Selbstständigen und Crowdworkern. Beitrag im Rahmen des 27. wissenschaftlichen Kongresses der Deutschen Vereinigung für Politikwissenschaft. Panel: Zukunft der Gewerkschaften in der Demokratie und der Demokratie in den Gewerkschaften. Verfügbar unter: https:/www.sowi.ruhr-uni-bochum.de/mam/content/sozomm/ kramer_2018_interessenvertretung_in_der_wolke_panel-beitrag_dvpw-kongress .pdf [07.06.2019].

Kupferschmitt, T. (2017). Onlinevideo: Gesamtreichweite stagniert, aber Streamingdienste punkten mit Fiction bei Jüngeren: Ergebnisse der ARD/ZDF-Onlinestudie 2017. Media Perspektiven (9), 447-462.

Lee, H., \& Staples, R. (2018). Digitale Solidarität unter Arbeitnehmer*innen. Industrielle Beziehungen. Zeitschrift für Arbeit, Organisation und Management, 25(4), 495-517.

Lehdonvirta, V., Kässi, O., Hjorth, I., Barnard, H., \& Graham, M. (2018). The Global Platform Economy: A New Offshoring Institution Enabling EmergingEconomy Microproviders. Journal of Management, 45(2), 567-599.

Leimeister, J. M., Durward, D., \& Zogaj, S. (2016). Crowd Worker in Deutschland: Eine empirische Studie zum Arbeitsumfeld auf externen Crowdsourcing-Plattformen (Study Nr. 323). Düsseldorf: Hans-Böckler-Stiftung. Verfügbar unter: https://www.boeckler.de/pdf/p_study_hbs_323.pdf [16.05.2019].

Lorig, P. (2015). Soloselbstständige Internet-Dienstleister im Niedriglohnbereich: Prekäres Unternehmertum auf Handwerksportalen im Spannungsfeld zwischen Autonomie und radikaler Marktabhängigkeit. Arbeits- und Industriesoziologische Studien, 8(1), 55-75.

Müller, A. (2016). The digital nomad: Buzzword or research category? Transnational Social Review, 6(3), 344-348.

Nash, C., Jarrahi, M. H., Sutherland, W., \& Phillips, G. (2018). Digital Nomads Beyond the Buzzword: Defining Digital Nomadic Work and Use of Digital Technologies. In G. Chowdhury, J. McLeod, V. Gillet \& P. Willett (Hrsg.), Lecture Notes in Computer Science: Bd. 10766. Transforming Digital Worlds: 13th International Conference, iConference 2018, Sheffield, UK, March 25-28, 2018, Proceedings (Bd. 10766, S. 207-217). Cham: Springer International Publishing. 
Niebler, V. (2019). Organizing YouTube. Rosa-Luxemburg-Stiftung. https:/www.r osalux.de/news/id/41394/organizing-youtube/ [17.12.2019].

Palmer, G. (2017). Foodora \& Co.: Die Revolte der neuen Dienstbotenklasse. Blätter für deutsche und internationale Politik (7), 29-32.

Papsdorf, C. (2018). Arbeit und Internet. In F. Böhle, G. G. Voß \& G. Wachtler (Hrsg.), Handbuch Arbeitssoziologie: Bd 1: Arbeit, Strukturen und Prozesse (2. Auflage) (S. 401-434). Wiesbaden: Springer.

Pesole, A., Urzí Brancati, C., Fernández-Macías, E., Biagi, F., \& González Vázquez, I. (2018). Platform workers in Europe: Evidence from the COLLEEM survey. JRC science for policy report. Publications Office of the European Union.

Pongratz, H. J., \& Bormann, S. (2017). Online-Arbeit auf Internet-Plattformen: Empirische Befunde zum 'Crowdworking' in Deutschland. Arbeits- und Industriesoziologische Studien, 10(2), 158-181.

Reichert, R. (2008). Amateure im Netz: Selbstmanagement und Wissenstechnik im Web 2.0. Bielefeld: transcript.

Schenk, M., Niermann, J., \& Briehl, A. (2014). Blogger 2014: Das Selbstverständnis von Themenbloggern und ihr Verhältnis zum Journalismus. Eine Studie im Auftrag des Deutschen Fachjournalisten-Verbands (DFJV). Verfügbar unter: https:/www.dfjv.de/documents/10180/178294/DFJV_Studie_Das_Selbstverstaen dnis_von_Themenbloggern.pdf [03.08.2018].

Schmidt, F. A. (2016). Arbeitsmärkte in der Plattformökonomie - Zur Funktionsweise und den Herausforderungen von Crowdwork und Gigwork. Bonn: Abteilung Wirtschafts- und Sozialpolitik der Friedrich-Ebert-Stiftung.

Schmidt, J.-H. (2006). Weblogs: Eine kommunikationssoziologische Studie. Kommunikationswissenschaft. Konstanz: UVK.

Schmidt, J.-H. (2018). Social Media (2. Auflage). Wiesbaden: Springer VS.

Schönefeld, D., \& Hensel, I. (2019). Autonomie und Kontrolle - Crowdworking "im Dazwischen": Einführung in den Sammelband. In I. Hensel, D. Schönefeld, E. Kocher, A. Schwarz \& J. Koch (Hrsg.), Selbstständige Unselbstständigkeit: Crowdworking zwischen Autonomie und Kontrolle (S.11-39). Baden-Baden: Nomos.

Hensel, I., Koch, J., Kocher, E., \& Schwarz, A. (2016). Crowdworking als Phänomen der Koordination digitaler Erwerbsarbeit - Eine interdisziplinäre Perspektive. Industrielle Beziehungen, 23(2), 162-186.

Schramm, F., \& Tietgen-Simonsen, M. (2019). Crowdworking - Menetekel und empirische Evidenz. In H. Hanau \& W. Matiaske (Hg.), Entgrenzung von Arbeitsverhältnissen: Arbeitsrechtliche und sozialwissenschaftliche Perspektiven (S. 11-24). Baden-Baden: Nomos.

Serfling, O. (2018). Crowdworking Monitor No. 1: für das Verbundprojekt "Crowdworking Monitor". Verfügbar unter: https://www.bmas.de/SharedDocs/ Downloads/DE/PDF-Meldungen/2018/crowdworking-monitor.pdf?_blob=publ icationFile\&v=1 https://doi.org/10.13140/RG.2.2.26833.79206 [27.02.2019]. 
Silberman, M. S., \& Irani, L. (2016). Operation an employer reputation system: Lessons from turkopticon, 2008-2015. Comparative Labor Law \& Policy Journal, $37(3), 505-542$.

Strube, S. (2015). Die Entstehung des digitalen Prekariats. Standpunkte 02/2015. Berlin: Rosa-Luxemburg-Stiftung. Verfügbar unter: https:/www.rosalux.de/publika tion/id/6734/die-entstehung-des-digitalen-prekariats [16.11.2020].

Taddicken, M., \& Schmidt, J.-H. (2017). Entwicklung und Verbreitung sozialer Medien. In J.-H. Schmidt \& M. Taddicken (Hrsg.), Handbuch Soziale Medien (S.3-22). Wiesbaden: Springer.

Urzí Brancati, M.C., Pesole, A., \& Fernández-Macías, E. (2020). New evidence on platform workers in Europe: Results from the second COLLEEM survey. JRC Science for Policy Report. Luxemburg: Publications Office of the European Union.

Vandaele, K. (2018). Will trade unions survive in the platform economy? Emerging patterns of platform workers' collective voice and representation in Europe (Working Paper). Brüssel: European Trade Union Institute (ETUI).

Ver.di. (2012). Crowdsourcing und Cloudworking: Gefahren für Gesellschaft und Arbeitnehmerinnen und Arbeitnehmer: Berliner Crowdsourcing-Cloudworking-Papier. Berlin: ver.di. Verfügbar unter: https://innovation-gute-arbeit.verdi. de/++file++557583e5aa698e5a58000e47/download/verdi-Positionspapier_Cloud working-Crowdsourcing.pdf [08.05.2019].

Wedde, P. (2017). Beschäftigtendatenschutz in der digitalisierten Welt (WISO Diskurs 09/2017). Bonn: Friedrich-Ebert-Stiftung.

Zander, I. (2018). Naturtalent der Mitbestimmung. MITBESTIMMUNG (07). Verfügbar unter: https://www.boeckler.de/de/magazin-mitbestimmung-2744-naturt alent-der-mitbestimmung-5844.htm [22.10.2020]. 